\title{
Nonresonance and Resonance Problems for Nonlocal Elliptic Equations with Respect to the Fučik Spectrum
}

\author{
Sarika Goyal
}

Abstract. In this article, we consider the following problem

$$
\begin{cases}(-\Delta)^{s} u=\alpha u^{+}-\beta u^{-}+f(u)+h & \text { in } \Omega \\ u=0 & \text { on } \mathbb{R}^{n} \backslash \Omega,\end{cases}
$$

where $\Omega \subset \mathbb{R}^{n}$ is a bounded domain with Lipschitz boundary, $n>2 s, 0<s<1$, $(\alpha, \beta) \in \mathbb{R}^{2}, f: \mathbb{R} \rightarrow \mathbb{R}$ is a bounded and continuous function and $h \in L^{2}(\Omega)$. We prove the existence results in two cases: first, the nonresonance case where $(\alpha, \beta)$ is not an element of the Fučik spectrum. Second, the resonance case where $(\alpha, \beta)$ is an element of the Fučik spectrum. Our existence results follows as an application of the saddle point theorem. It extends some results, well known for Laplace operator, to the nonlocal operator.

\section{Introduction}

Let $\Omega \subset \mathbb{R}^{n}$ be a bounded domain with Lipschitz boundary, $n>2 s$ and $s \in(0,1)$. Then consider the following problem

$$
\begin{cases}(-\Delta)^{s} u=\alpha u^{+}-\beta u^{-}+f(u)+h & \text { in } \Omega \\ u=0 & \text { on } \mathbb{R}^{n} \backslash \Omega,\end{cases}
$$

where $(\alpha, \beta) \in \mathbb{R}^{2}, u^{ \pm}=\max \{ \pm u, 0\}, f: \mathbb{R} \rightarrow \mathbb{R}$ is a bounded and continuous function, $h \in L^{2}(\Omega)$ and $(-\Delta)^{s}$ is the fractional Laplacian operator defined as

$$
(-\Delta)^{s} u(x)=-\frac{1}{2} \int_{\mathbb{R}^{n}} \frac{u(x+y)+u(x-y)-2 u(x)}{|y|^{n+2 s}} d y \text { for all } x \in \mathbb{R}^{n} .
$$

In this article, we study the corresponding problem driven by the nonlocal operator $\mathcal{L}_{K}$ given by

$$
\begin{cases}-\mathcal{L}_{K} u=\alpha u^{+}-\beta u^{-}+f(u)+h & \text { in } \Omega, \\ u=0 & \text { on } \mathbb{R}^{n} \backslash \Omega .\end{cases}
$$

Received May 30, 2019; Accepted February 25, 2020.

Communicated by Jann-Long Chern.

2010 Mathematics Subject Classification. 35A15, 35B33, 35H39.

Key words and phrases. nonlocal problem, Fučik spectrum, resonance, nonresonance, saddle point theorem. 
The nonlocal operator $\mathcal{L}_{K}$ is defined as

$$
\mathcal{L}_{K} u(x):=-\frac{1}{2} \int_{\mathbb{R}^{n}}(u(x+y)+u(x-y)-2 u(x)) K(y) d y \quad \text { for all } x \in \mathbb{R}^{n},
$$

and the function $K: \mathbb{R}^{n} \backslash\{0\} \rightarrow(0, \infty)$ satisfies the following assumptions:

(K1) $m K \in L^{1}\left(\mathbb{R}^{n}\right)$, where $m(x)=\min \left\{|x|^{2}, 1\right\}$,

(K2) There exist $\lambda>0$ and $s \in(0,1)$ such that $K(x) \geq \lambda|x|^{-(n+2 s)}$,

(K3) $K(x)=K(-x)$ for any $x \in \mathbb{R}^{n} \backslash\{0\}$.

In case $K(x)=|x|^{-(n+2 s)}, \mathcal{L}_{K}$ is the fractional Laplace operator $(-\Delta)^{s}$. When $s=1$, the fractional Laplacian operator becomes the usual Laplace operator. There has been done a lot of works related to the solvability of resonance problem with respect to the Fučik spectrum for the classical Laplace equation, see [7,8, 13, 14 and references therein. The Fučik spectrum in the case of Laplacian and $p$-Laplacian equations with Dirichlet boundary condition has been studied by many authors [4,5, 10].

Recently a lot of attention has been paid to the study of fractional and nonlocal equations of elliptic type due to concrete real world applications in finance, thin obstacle problem, optimization, quasi-geostrophic flow etc., see [1,2,18]. Dirichlet boundary value problem in case of fractional Laplacian with polynomial type nonlinearity using variational methods is studied in [15,16]. Fiscella, Servadei and Valdinoci in [11] studied the resonance problem with respect to the spectrum for a non local equation.

The Fučik spectrum of the nonlocal operator $\mathcal{L}_{K}$ is defined as the set

$$
\Sigma_{K}:=\left\{(\alpha, \beta) \in \mathbb{R}^{2} \mid-\mathcal{L}_{K} u=\alpha u^{+}-\beta u^{-} \text {in } \Omega, u=0 \text { on } \mathbb{R}^{n} \backslash \Omega\right\},
$$

where $u$ is a nontrivial solution. For $\alpha=\beta=\lambda$, the Fučik spectrum of 1.2 becomes the usual spectrum of $\mathcal{L}_{K}$. In this case, $u$ satisfies

$$
\begin{cases}-\mathcal{L}_{K} u=\lambda u & \text { in } \Omega \\ u=0 & \text { on } \mathbb{R}^{n} \backslash \Omega .\end{cases}
$$

Let $0<\lambda_{1}<\lambda_{2} \leq \cdots \leq \lambda_{k} \leq \cdots$ denote the sequence of eigenvalues of $(1.3)$ and $\left\{\phi_{k}\right\}_{k}$ denote the sequence of eigenfunctions corresponding to $\lambda_{k}$. Then it is proved in [16 that the first eigenvalue $\lambda_{1}$ of $(1.3)$ is simple, isolated and can be characterized as follows:

$$
\lambda_{1}=\inf _{u \in X_{0}}\left\{\int_{Q}(u(x)-u(y))^{2} K(x-y) d x d y: \int_{\Omega} u^{2}=1\right\} .
$$

The authors also proved that the eigenfunctions corresponding to $\lambda_{1}$ is non-negative. Also such eigenfunction is strictly positive in $\Omega$, thanks to [17]. Moreover, one can observe 
that $\Sigma_{K}$ clearly contains $\left(\lambda_{k}, \lambda_{k}\right)$ for each $k \in \mathbb{N}$ and $\Sigma_{K}$ is symmetric with respect to the diagonal. In [12], it is shown that the two lines $\mathbb{R} \times \lambda_{1}$ and $\lambda_{1} \times \mathbb{R}$ belongs to $\Sigma_{K}$ and are isolated in $\Sigma_{K}$. Also a variational characterization of the second eigenvalue $\lambda_{2}$ of $-\mathcal{L}_{K}$ is studied in [12]. But here we will characterize a portion of $\Sigma_{K}$ using the variational methods. That is, the eigenvalue pair will be obtained as minima or minimax values of an appropriate functional.

In the homogeneous case where $\alpha=\beta=\lambda$ and $f \equiv 0$, the solvability of 1.1 can be completely described by the Fredholm Alternative, which says that if $\lambda$ is not an eigenvalue of $-\mathcal{L}_{K}$, then the problem has a unique solution for any $h$, and if $\lambda$ is an eigenvalue of $-\mathcal{L}_{K}$, then the problem 1.1 has a solution if and only if $h$ is orthogonal to the corresponding eigenspace.

For the nonhomogeneous case where $\alpha=\beta=\lambda$ and $f \neq 0$, Fiscella, Servadei and Valdinoci in [11], studied the existence results for the following problem

$$
\begin{cases}-\mathcal{L}_{K} u+q(x) u=\lambda u+f(u)+h(x) & \text { in } \Omega \\ u=0 & \text { on } \mathbb{R}^{n} \backslash \Omega\end{cases}
$$

where $f, q$ and $h$ are sufficiently smooth functions. They showed that if $\lambda$ is not an eigenvalue (nonresonance), then it has a solution with no further restriction on $f$ and $h$, and if $\lambda$ is an eigenvalue (resonance), then they need some extra conditions on $f$ and $h$. Precisely, denoting by

$$
f_{l}=\lim _{t \rightarrow-\infty} f(t) \quad \text { and } \quad f_{r}=\lim _{t \rightarrow \infty} f(t)
$$

they assume that $f_{l}$ and $f_{r}$ exist, are finite and such that $f_{l}>f_{r}$ and

$$
f_{r} \int_{\Omega} \phi^{-}(x) d x-f_{l} \int_{\Omega} \phi^{+}(x) d x<\int_{\Omega} h(x) \phi(x) d x<f_{l} \int_{\Omega} \phi^{-}(x) d x-f_{r} \int_{\Omega} \phi^{+}(x) d x
$$

for any nontrivial $\phi$ in the eigenspace associated with $\lambda$. We would remark that these extra conditions on $f$ and $h$ are exactly the same required in the resonant setting, when dealing with the classical Laplace operator. Moreover, in the resonant case for fractional Laplacian, they are able to treat this case only if $\lambda$ satisfies the following condition:

$\lambda$ is an eigenvalue of $-\mathcal{L}_{K}+q$ such that all the eigenfunctions corresponding to $\lambda$ have nodal set with zero Lebesgue measure.

As usual, the nodal set of a function $g$ in $\Omega$ is the level set $\{x \in \Omega: g(x)=0\}$. The condition (1.4) has been established for the fractional Laplacian in [9]. For instant, in case of fractional Laplacian (1.4) is true when $\lambda$ is its first eigenvalue however condition (1.4) is satisfied by every eigenvalue of the classical Laplace operator. At this point, we note that 
there is a difference in result between Nonlocal operator and Classical Laplcian operator. Therefore one can not directly extend the results for Laplace equation to nonlocal equation due to the nonlocal behaviour of the operator and the bounded support of the test function is not preserved.

In this paper, we studied the problem (1.1) with respect to the Fučik spectrum for nonlocal equation. Here we use the variational argument which was developed by Castro and Chang in [4] for the Laplace operator. To the best of our knowledge, no work has been done related to the solvability of resonance and nonresonance problems with respect to the Fučik spectrum for the nonlocal equation in (1.1). The results obtained here are somehow expected but new for the nonlocal operator.

Now for the nonresonance case, we assume that $\alpha$ lies strictly between consecutive eigenvalues of $(-\Delta)^{s}$, call them as $\lambda_{k}<\lambda_{k+1}$, and we also assume that $\alpha \leq \beta<\beta(\alpha)$, where $\beta(\alpha)$ is defined as

$$
\beta(\alpha):=\sup \left\{\beta \geq \alpha \mid \widetilde{J}_{\alpha, b}(y)=J_{\alpha, b}\left(y+M_{\alpha, b}(y)\right)>0 \text { for all } b \in(\alpha, \beta), y \in X_{2} \backslash\{0\}\right\},
$$

where $J_{\alpha, b}$ is a function defined in (2.2) and $\{(\alpha, \beta): \alpha \leq \beta<\beta(\alpha)\}$ contains no points in $\Sigma_{K}$, according to the Castro-Chang characterization (see Theorem 2 in [4]) which can be easily generalized in case of fractional Laplace operator and it is stated as follows:

Proposition 1.1. If $\alpha \in\left(\lambda_{k}, \lambda_{k+1}\right), N(l)<\infty$ (multiplicity of eigenvalue $\lambda_{l}$ ) for $l \geq k+1$, and $\beta(\alpha)$ is defined as above. Then the following hold.

(1) $(\alpha, \beta(\alpha))$ is in the Fučik spectrum when $\beta(\alpha)<+\infty$.

(2) If $\beta \in[\alpha, \beta(\alpha))$ then $(\alpha, \beta)$ is not in the Fučik spectrum.

(3) For $\beta>\alpha,(\alpha, \beta)$ is in the Fučik spectrum if and only if the restriction of $\widetilde{J}_{\alpha, \beta}$ to $\left\{y \in X_{2} \mid\|y\|=1\right\}$ has a critical point on $\left\{y \in X_{2} \mid\|y\|=1, \widetilde{J}_{\alpha, \beta}=0\right\}$.

(4) The function $\beta(\alpha):\left(\lambda_{j}, \lambda_{j+1}\right) \rightarrow[0,+\infty], \alpha \rightarrow \beta(\alpha)$ is non-increasing and continuous.

Note that if $X_{2} \backslash\{0\}$ contains a non-negative function then $\beta(\alpha)<\infty$ for all $\alpha \in\left(\lambda_{k}, \lambda_{k+1}\right)$.

In the prove of above theorem, one use the following global reduction principle (see [3]).

Proposition 1.2. Let $H$ be a separable real Hilbert space. Let $X_{1}, X_{2}$ be closed subspaces such that $H=X_{1} \oplus X_{2}$, and $J: H \rightarrow \mathbb{R}$ a functional of class $C^{1}$. If there exists $m>0$ such that

$$
\left\langle\nabla J\left(x_{1}+x_{2}\right)-\nabla J\left(y_{1}+x_{2}\right), x_{1}-y_{1}\right\rangle \leq-m\left\|x_{1}-y_{1}\right\|^{2}
$$

for all $x_{1}, y_{1} \in X_{1}, x_{2} \in X_{2}$, then there exists a continuous function $M: X_{2} \rightarrow X_{1}$ such that 
(1) $J(y+M(y))=\max \left\{J(y+x) \mid x \in X_{1}\right\}$.

(2) $\widetilde{J}: Y \rightarrow \mathbb{R}$ defined by $\widetilde{J}(y)=J(y+M(y))$ is of class $C^{1}$.

(3) $x+y$ is a critical point of $J$ if and only if $x=M(y)$ and $y$ is a critical point of $\widetilde{J}$.

Now we state the main results.

Theorem 1.3 (Nonresonance case). Assume $\lambda_{k}<\alpha<\lambda_{k+1}, \alpha \leq \beta<\beta(\alpha), f: \mathbb{R} \rightarrow \mathbb{R}$ is a bounded and continuous function, and $h \in L^{2}(\Omega)$, then the problem (1.1) has at least one weak solution.

In the resonance case, we still assume that $\lambda_{k}<\alpha<\lambda_{k+1}$, but now assume that $\beta=\beta(\alpha)$, as above, where $(\alpha, \beta(\alpha)) \in \Sigma_{K}$. The solvability condition that we impose is the following:

Let $F(u):=\int_{0}^{u} f(t) d t$. If $\left\{u_{k}\right\} \in X_{0}$ (see (2.1)), is such that $\left\|u_{k}\right\|_{L^{2}} \rightarrow \infty$ and $\frac{u_{k}}{\left\|u_{k}\right\|_{L^{2}}}$ converges in $L^{2}(\Omega)$ to some $v$, a nontrivial Fučik eigenfunction associated with $(\alpha, \beta)$, then $\lim _{k \rightarrow \infty} \int_{\Omega}\left(F\left(u_{k}\right)+h u_{k}\right) d x=-\infty$. Moreover, this

$$
\lim _{k \rightarrow \infty} \int_{\Omega}\left(F\left(u_{k}\right)+h u_{k}\right) d x=-\infty
$$

is known as the generalization of Landesman-Lazer condition. The following theorem is the boarder line case comparing to Theorem 1.3 .

Theorem 1.4 (Resonance case). Assume $\lambda_{k}<\alpha<\lambda_{k+1}, \beta=\beta(\alpha), f: \mathbb{R} \rightarrow \mathbb{R}$ is bounded and continuous satisfying $\lim _{k \rightarrow \infty} \int_{\Omega}\left(F\left(u_{k}\right)+h u_{k}\right) d x=-\infty$, where $F$ is primitive of $f$ and $h \in L^{2}(\Omega)$. Then the problem (1.1) has at least one weak solution.

\section{Preliminaries}

In this section we will recall function spaces which was introduced by Servadei and Valdinoci in 15, 16 and some standard results from Functional analysis and critical point Theory.

Now due to nonlocalness of the fractional Laplacian, we define the function spaces introduced by Servadei and Valdinoci in [15] as

$$
X=\left\{u \mid u: \mathbb{R}^{n} \rightarrow \mathbb{R} \text { is measurable, }\left.u\right|_{\Omega} \in L^{2},(u(x)-u(y)) \sqrt{K(x-y)} \in L^{2}(Q)\right\},
$$

where $Q=\mathbb{R}^{2 n} \backslash(\mathcal{C} \Omega \times \mathcal{C} \Omega)$ and $\mathcal{C} \Omega:=\mathbb{R}^{n} \backslash \Omega$. The space $X$ is endowed with the norm $\|u\|_{X}=\|u\|_{L^{2}(\Omega)}+\left(\int_{Q}|u(x)-u(y)|^{2} K(x-y) d x d y\right)^{1 / 2}$. Then we define

$$
X_{0}=\left\{u \in X: u=0 \text { a.e. in } \mathbb{R}^{n} \backslash \Omega\right\}
$$


equipped with the norm

$$
\|u\|=\left(\int_{Q}|u(x)-u(y)|^{2} K(x-y) d x d y\right)^{1 / 2},
$$

is a Hilbert spaces. Note that the norm $\|\cdot\|$ on the space $X_{0}$ involves the interaction between $\Omega$ and $\mathbb{R}^{n} \backslash \Omega$. For more details on these function spaces and the embedding theorems, we refer to 6,15 .

We also recall the space $L^{2}(\Omega):=\left\{u: \Omega \rightarrow \mathbb{R}: u\right.$ is measurable, $\left.\int_{\Omega} u^{2} d x<\infty\right\}$ endowed with the norm $\|u\|_{L^{2}}=\left(\int_{\Omega} u^{2} d x\right)^{1 / 2}$ is a Hilbert space.

Definition 2.1. A function $u \in X_{0}$ is a weak solution of (1.1), if for every $v \in X_{0}, u$ satisfies

$$
\int_{Q}(u(x)-u(y))(v(x)-v(y)) K(x-y) d x d y=\int_{\Omega}\left(\alpha u^{+}-\beta u^{-}+f(u)+h\right) v d x .
$$

Now we denote $X_{1}:=\operatorname{span}\left[\phi_{1}, \phi_{2}, \ldots, \phi_{k}\right]$. That is, the linear span of the first $k$ eigenfunctions, and $X_{2}:=X_{1}^{\perp}=\left[\phi_{k+1}, \phi_{k+2}, \ldots\right]$. The sequence $\left\{\phi_{k}\right\}_{k \in \mathbb{N}}$ of eigenfunctions is an orthonormal basis of $L^{2}(\Omega)$ and an orthogonal basis of $X_{0}$. By definition, the subspaces $X_{1}$ and $X_{2}$ are orthogonal and $X_{0}=X_{1} \oplus X_{2}$. The Fourier expansion of a function $u \in X_{0}$ is $u=\sum_{j=1}^{\infty} c_{j} \phi_{j}$. Then note that

$$
\int_{Q}|u(x)-u(y)|^{2} K(x-y) d x d y=\sum_{j=1}^{\infty} \lambda_{j} c_{j}^{2} \quad \text { and } \quad \int_{\Omega} u^{2} d x=\sum_{j=1}^{\infty} c_{j}^{2} .
$$

This has helpful consequence such as

$$
\begin{aligned}
& \int_{Q}|u(x)-u(y)|^{2} K(x-y) d x d y \leq \lambda_{k} \int_{\Omega} u^{2} d x, \quad \forall u \in X_{1}, \\
& \int_{Q}|v(x)-v(y)|^{2} K(x-y) d x d y \geq \lambda_{k+1} \int_{\Omega} v^{2} d x, \quad \forall v \in X_{2} .
\end{aligned}
$$

To analyze problem 1.2 , we consider the functional

$$
J_{\alpha, \beta}(u)=\frac{1}{2}\left(\int_{Q}|u(x)-u(y)|^{2} K(x-y) d x d y-\alpha \int_{\Omega}\left(u^{+}\right)^{2} d x-\beta \int_{\Omega}\left(u^{-}\right)^{2} d x\right),
$$

which is $C^{1}$ functional on $X_{0}$ with

$$
\left\langle J_{\alpha, \beta}^{\prime}(u), v\right\rangle=\int_{Q}(u(x)-u(y))(v(x)-v(y)) K(x-y) d x d y-\alpha \int_{\Omega} u^{+} v d x+\beta \int_{\Omega} u^{-} v d x .
$$

One can easily see that the critical points of $J_{\alpha, \beta}$ are weak solutions of 1.2 . It will be very useful to think of $J_{\alpha, \beta}$ as a $C^{1}$ functional on $\mathbb{R}^{2} \times X_{0}$. That is,

$$
J: \mathbb{R}^{2} \times X_{0} \rightarrow \mathbb{R}, \quad J(\alpha, \beta, u):=J_{\alpha, \beta}(u)
$$


with the derivative given by

$$
\begin{aligned}
\left\langle J^{\prime}(\alpha, \beta, u),(s, t, v)\right\rangle= & \int_{Q}(u(x)-u(y))(v(x)-v(y)) K(x-y) d x d y \\
& -\alpha \int_{\Omega} u^{+} v+\beta \int_{\Omega} u^{-} v-s \int_{\Omega}\left(u^{+}\right)^{2}-t \int_{\Omega}\left(u^{-}\right)^{2} .
\end{aligned}
$$

It is clear that $\|D J\|_{\left(\mathbb{R}^{2} \times X_{0}\right)^{*}}$ is bounded on bounded subsets of $\mathbb{R}^{2} \times X_{0}$, and so $J$ is uniformly Lipschitz continuous on any bounded subset of $\mathbb{R}^{2} \times X_{0}$.

To examine problem (1.1), we consider the functional

$$
E_{\alpha, \beta}(u)=\frac{1}{2}\left(\int_{Q}|u(x)-u(y)|^{2} K(x-y) d x d y-\alpha \int_{\Omega}\left(u^{+}\right)^{2}-\beta \int_{\Omega}\left(u^{-}\right)^{2}\right)-\int_{\Omega}(F(u)+h u),
$$

where $F(u):=\int_{0}^{u} f(t) d t . E_{\alpha, \beta}$ is also a $C^{1}$ functional on $X_{0}$ with

$$
\left\langle E_{\alpha, \beta}^{\prime}(u), v\right\rangle=\int_{Q}(u(x)-u(y))(v(x)-v(y)) K(x-y) d x d y-\int_{\Omega}\left(\alpha u^{+}-\beta u^{-}+f(u)+h\right) v d x .
$$

It is straightforward to see that critical points of $E_{\alpha, \beta}$ are weak solutions of (1.1).

We will use the saddle point theorem to prove the existence of critical points. Before stating the theorem, we first define the Palais-Smale condition (PS).

Definition 2.2. Let $J: H \rightarrow \mathbb{R}$ be a $C^{1}$ functional on a Banach space $H$. Then we say that $J$ satisfies (PS) if for any sequence $\left\{u_{k}\right\} \subset H$ such that $J\left(u_{k}\right)$ is bounded and $J^{\prime}\left(u_{k}\right) \rightarrow 0$ in $H^{*}$, there is a converging subsequence of $\left\{u_{k}\right\}$.

Theorem 2.3 (Saddle Point Theorem). Let $J: X_{0} \rightarrow \mathbb{R}$ be a $C^{1}$ functional which satisfies Palais-Smale (PS) condition. Assume that there are sets $\mathcal{X}_{1}, \mathcal{X}_{2} \subset X_{0}$ such that

(i) $\mathcal{X}_{1}=\widetilde{\gamma}\left(S^{k-1}\right)$, where $\widetilde{\gamma}: S^{k-1} \rightarrow X_{0}$ is continuous.

(ii) $\mathcal{X}_{2}$ links with $\mathcal{X}_{1}$, i.e., if $B$ is the unit ball in $\mathbb{R}^{k}$ and $\gamma: B \rightarrow X_{0}$ is a continuous function such that $\gamma \equiv \widetilde{\gamma}$ on $S^{k-1}$, then $\gamma(B) \cap \mathcal{X}_{2} \neq \emptyset$.

(iii) $\sup _{x \in \mathcal{X}_{1}} J(x)<\inf _{y \in \mathcal{X}_{2}} J(y)$.

Then $c:=\inf _{\gamma \in \Gamma} \sup _{x \in B} J(\gamma(x))$ is a critical point of $J$, where $\Gamma=\left\{\gamma: B \rightarrow X_{0}\right.$ : $\gamma$ is continuous and $\gamma \equiv \widetilde{\gamma}$ on $\left.S^{k-1}\right\}$.

\section{The variational characterization of Fučik spectrum}

In all that follows we assume that $\lambda_{k}<\alpha<\lambda_{k+1}$ and the points of $\Sigma_{K}$ that we characterize will all lie in this vertical strip in the $(\alpha, \beta)$ plane. We assume that $\alpha \leq \beta$, and note that opposite case can be treated via symmetric arguments. Our approach to find the critical points of $J_{\alpha, \beta}$ will take advantage of concavity to maximize in the $X_{1}$ direction, and then to use weak lower semicontinuity to minimize in the $X_{2}$ direction. 


\subsection{Maximizing in the $X_{1}$ direction}

In this subsection, we will show that the functional $J_{\alpha, \beta}$ attains a maximizer in the $X_{1}$ direction and the properties of the maximizer function. First, we prove the general inequality that is used to prove the concavity of the functional in $X_{1}$ direction.

Lemma 3.1. Let $\left(\alpha_{i}, \beta_{i}\right) \in \mathbb{R}^{2}$ for $i=1,2$, be points satisfying $\alpha_{i} \leq \beta_{i}$, and let $s_{i}=\beta_{i}-\alpha_{i}$. Let $u_{i} \in X_{1}$ and $v_{i} \in X_{2}$ for $i=1,2$. Then there exist $a \delta=\alpha_{2} / \lambda_{k}-1>0$ such that

$$
\begin{aligned}
& \left\langle\left(J_{\alpha_{2}, \beta_{2}}^{\prime}\left(u_{2}+v_{2}\right)-J_{\alpha_{1}, \beta_{1}}^{\prime}\left(u_{1}+v_{1}\right)\right),\left(u_{2}-u_{1}\right)\right\rangle \\
\leq & -\delta\left\|u_{2}-u_{1}\right\|^{2}+\left|\beta_{2}-\alpha_{2}\right|\left(\left\|u_{2}-u_{1}\right\|_{L^{2}}+\left\|v_{2}-v_{1}\right\|_{L^{2}}\right)\left\|v_{2}-v_{1}\right\|_{L^{2}} \\
& +\left|\alpha_{2}-\alpha_{1}\right|\left\|u_{1}\right\|_{L^{2}}\left\|u_{2}-u_{1}\right\|_{L^{2}}+\left|s_{2}-s_{1}\right|\left\|u_{1}+v_{1}\right\|_{L^{2}}\left\|u_{2}-u_{1}\right\|_{L^{2}} .
\end{aligned}
$$

Proof. Consider

$$
\begin{aligned}
& \left\langle J_{\alpha_{i}, \beta_{i}}^{\prime}\left(u_{i}+v_{i}\right),\left(u_{2}-u_{1}\right)\right\rangle \\
= & \int_{Q}\left(\left(u_{i}+v_{i}\right)(x)-\left(u_{i}+v_{i}\right)(y)\right)\left(\left(u_{2}-u_{1}\right)(x)-\left(u_{2}-u_{1}\right)(y)\right) K(x-y) d x d y \\
& -\alpha_{i} \int_{\Omega}\left(u_{i}+v_{i}\right)^{+}\left(u_{2}-u_{1}\right)+\beta_{i} \int_{\Omega}\left(u_{i}+v_{i}\right)^{-}\left(u_{2}-u_{1}\right) \\
= & \int_{Q}\left(\left(u_{i}+v_{i}\right)(x)-\left(u_{i}+v_{i}\right)(y)\right)\left(\left(u_{2}-u_{1}\right)(x)-\left(u_{2}-u_{1}\right)(y)\right) K(x-y) d x d y \\
& -\alpha_{i} \int_{\Omega}\left(u_{i}+v_{i}\right)\left(u_{2}-u_{1}\right)+s_{i} \int_{\Omega}\left(u_{i}+v_{i}\right)^{-}\left(u_{2}-u_{1}\right) .
\end{aligned}
$$

Then using the orthogonality of $X_{1}$ and $X_{2}$, we obtain

$$
\begin{aligned}
& \left\langle J_{\alpha_{i}, \beta_{i}}^{\prime}\left(u_{i}+v_{i}\right),\left(u_{2}-u_{1}\right)\right\rangle \\
= & \int_{Q}\left(u_{i}(x)-u_{i}(y)\right)\left(\left(u_{2}-u_{1}\right)(x)-\left(u_{2}-u_{1}\right)(y)\right) K(x-y) d x d y \\
& -\alpha_{i} \int_{\Omega} u_{i}\left(u_{2}-u_{1}\right)+s_{i} \int_{\Omega}\left(u_{i}+v_{i}\right)^{-}\left(u_{2}-u_{1}\right) .
\end{aligned}
$$

Subtracting the above expression for $i=1,2$ gives

$$
\begin{aligned}
& \left\langle\left(J_{\alpha_{2}, \beta_{2}}^{\prime}\left(u_{2}+v_{2}\right)-J_{\alpha_{1}, \beta_{1}}^{\prime}\left(u_{1}+v_{1}\right)\right),\left(u_{2}-u_{1}\right)\right\rangle \\
= & \int_{Q}\left|\left(u_{2}-u_{1}\right)(x)-\left(u_{2}-u_{1}\right)(y)\right|^{2} K(x-y) d x d y-\int_{\Omega}\left(\alpha_{2} u_{2}-\alpha_{1} u_{1}\right)\left(u_{2}-u_{1}\right) \\
& +\int_{\Omega}\left(s_{2}\left(u_{2}+v_{2}\right)^{-}-s_{1}\left(u_{1}+v_{1}\right)^{-}\right)\left(u_{2}-u_{1}\right) \\
= & \left\|u_{2}-u_{1}\right\|^{2}-\alpha_{2} \int_{\Omega}\left|u_{2}-u_{1}\right|^{2}+s_{2} \int_{\Omega}\left(\left(u_{2}+v_{2}\right)^{-}-\left(u_{1}+v_{1}\right)^{-}\right)\left(u_{2}-u_{1}\right) \\
& +\left(s_{2}-s_{1}\right) \int_{\Omega}\left(u_{1}+v_{1}\right)^{-}\left(u_{2}-u_{1}\right)-\left(\alpha_{2}-\alpha_{1}\right) \int_{\Omega} u_{1}\left(u_{2}-u_{1}\right) .
\end{aligned}
$$


Now we analyze each term of the right hand side separately. First, it is clear from the definition of $X_{1}$ and the standard characterization of the eigenvalue of $(-\Delta)^{s}$ that

$$
\left\|u_{2}-u_{1}\right\|^{2}-\alpha_{2} \int_{\Omega}\left|u_{2}-u_{1}\right|^{2} \leq\left(1-\frac{\alpha_{2}}{\lambda_{k}}\right)\left\|u_{2}-u_{1}\right\|^{2}=-\delta\left\|u_{2}-u_{1}\right\|^{2} .
$$

From the Hölder's inequality, we obtain

$$
\int_{\Omega} u_{1}\left(u_{2}-u_{1}\right) \leq\left\|u_{1}\right\|_{L^{2}}\left\|u_{2}-u_{1}\right\|_{L^{2}}
$$

Using the relation $f=f^{+}-f^{-}$, the monotonicity of $g(t)=t^{-}$, the fact that $\left|g\left(t_{1}\right)-g\left(t_{2}\right)\right| \leq$ $\left|t_{2}-t_{1}\right|$ and Hölder's inequality, we obtain

$$
\begin{aligned}
& s_{2} \int_{\Omega}\left(\left(u_{2}+v_{2}\right)^{-}-\left(u_{1}+v_{1}\right)^{-}\right)\left(u_{2}-u_{1}\right) \\
= & s_{2} \int_{\Omega}\left(\left(u_{2}+v_{2}\right)^{-}-\left(u_{1}+v_{1}\right)^{-}\right)\left(\left(u_{2}+v_{2}\right)-\left(u_{1}+v_{1}\right)\right) \\
& -s_{2} \int_{\Omega}\left(\left(u_{2}+v_{2}\right)^{-}-\left(u_{1}+v_{1}\right)^{-}\right)\left(v_{2}-v_{1}\right) \\
= & -s_{2} \int_{\Omega}\left[\left(\left(u_{2}+v_{2}\right)^{-}\right)^{2}+\left(\left(u_{1}+v_{1}\right)^{-}\right)^{2}+\left(u_{2}+v_{2}\right)^{+}\left(u_{1}+v_{1}\right)^{-}+\left(u_{2}+v_{2}\right)^{-}\left(u_{1}+v_{1}\right)^{+}\right] \\
& -s_{2} \int_{\Omega}\left(\left(u_{2}+v_{2}\right)^{-}-\left(u_{1}+v_{1}\right)^{-}\right)\left(v_{2}-v_{1}\right) \\
\leq & s_{2} \int_{\Omega}\left|\left(u_{2}-u_{1}\right)+\left(v_{2}-v_{1}\right) \| v_{2}-v_{1}\right| \\
\leq & s_{2}\left(\left\|u_{2}-u_{1}\right\|_{L^{2}}+\left\|v_{2}-v_{1}\right\|_{L^{2}}\right)\left\|v_{2}-v_{1}\right\|_{L^{2} .} .
\end{aligned}
$$

Combining the above inequalities together we obtain the desired result.

First, we recall the definition of anticoercivity.

Definition 3.2. A functional $J: X_{0} \rightarrow \mathbb{R}$ on a Banach space $X_{0}$ is called coercive if for every sequence $\left\{u_{k}\right\} \subset X_{0},\left\|u_{k}\right\| \rightarrow+\infty$ implies $J\left(u_{k}\right) \rightarrow+\infty$. A functional $J$ is called anticoercive if $-J$ is coercive.

Lemma 3.3. For every $v \in X_{2}$, the functional $J_{\alpha, \beta}(\cdot, v): X_{1} \rightarrow \mathbb{R}$ is strictly concave and anticoercive.

Proof. Taking $\alpha=\alpha_{2}=\alpha_{1}, \beta=\beta_{2}=\beta_{1}$ and $v_{2}=v_{1}=v$, in (3.1), we obtain

$$
\left\langle\left(J_{\alpha, \beta}^{\prime}\left(u_{2}+v\right)-J_{\alpha, \beta}^{\prime}\left(u_{1}+v\right)\right),\left(u_{2}-u_{1}\right)\right\rangle \leq-\delta\left\|u_{2}-u_{1}\right\|^{2},
$$

which implies strict concavity. Then, the anticoercivity of $J_{\alpha, \beta}$ now follows from the strict concavity and the Fundamental Theorem of Calculus. 
Lemma 3.4. For every $v \in X_{2}$, the functional $J_{\alpha, \beta}(\cdot, v): X_{1} \rightarrow \mathbb{R}$ achieves a unique maximum.

Proof. Let $\left\{u_{k}+v\right\}$ be a maximizing sequence. Then anticoercivity of $J_{\alpha, \beta}$ implies that the sequence $\left\{u_{k}\right\}$ is bounded in $X_{1}$. Therefore the sequence $\left\{u_{k}\right\}$ has a weakly convergent subsequence. Also $J_{\alpha, \beta}$ is weakly upper semicontinuous, follows from concavity of $J_{\alpha, \beta}$. So, $J_{\alpha, \beta}$ achieves its maximum. Uniqueness follows easily from the strict concavity.

Thus, the above result makes possible to define the definition.

Definition 3.5. The function $M_{\alpha, \beta}: X_{2} \rightarrow X_{1}$ is defined as

$$
M_{\alpha, \beta}(v)=\max _{u \in X_{1}} J_{\alpha, \beta}(u, v) .
$$

Now we investigate a few useful properties of $M_{\alpha, \beta}$. We start with homogeneity.

Lemma 3.6. If $t \geq 0$ and $v \in X_{2}$, then $M_{\alpha, \beta}(t v)=t M_{\alpha, \beta}(v)$.

Proof. Case 1: $t>0$. Then by the maximizing property of $M_{\alpha, \beta}$, we have

$$
J_{\alpha, \beta}\left(M_{\alpha, \beta}(t v)+t v\right) \geq J_{\alpha, \beta}(u+t v) \quad \text { for all } u \in X_{1} .
$$

Using the homogeneity of $J_{\alpha, \beta}$, we obtain that

$$
J_{\alpha, \beta}\left(\frac{M_{\alpha, \beta}(t v)}{t}+v\right) \geq J_{\alpha, \beta}\left(\frac{u}{t}+v\right) \quad \text { for all } u \in X_{1} .
$$

Hence

$$
J_{\alpha, \beta}\left(\frac{M_{\alpha, \beta}(t v)}{t}+v\right) \geq J_{\alpha, \beta}(u+v) \quad \text { for all } u \in X_{1} .
$$

Thus for any $t>0, M_{\alpha, \beta}(t v)=t M_{\alpha, \beta}(v)$.

Case 2: $t=0$. Then it only needs to argue that $M_{\alpha, \beta}(0)=0$. It is immediate that $J_{\alpha, \beta}(0)=0$. It suffices to show that $J_{\alpha, \beta}(u)<0$ for $u \in X_{1} \backslash\{0\}$. Recall that $\int_{Q}|u(x)-u(y)|^{2} K(x-y) d x d y \leq \lambda_{k} \int_{\Omega} u^{2} d x$ for all $u \in X_{1}$ and that $\lambda_{k}<\alpha<\beta$. It follows that

$$
\begin{aligned}
J_{\alpha, \beta}(u) & =\frac{1}{2}\left(\int_{Q}|u(x)-u(y)|^{2} K(x-y)-\alpha \int_{\Omega}\left(u^{+}\right)^{2} d x-\beta \int_{\Omega}\left(u^{-}\right)^{2} d x\right) \\
& \leq \frac{1}{2}\left(\lambda_{k} \int_{\Omega} u^{2} d x-\alpha \int_{\Omega}\left(u^{+}\right)^{2} d x-\beta \int_{\Omega}\left(u^{-}\right)^{2} d x\right) \\
& \leq \frac{1}{2}\left(\lambda_{k} \int_{\Omega} u^{2} d x-\alpha \int_{\Omega}\left(u^{+}\right)^{2} d x-\alpha \int_{\Omega}\left(u^{-}\right)^{2} d x\right) \\
& =\frac{1}{2}\left(\lambda_{k}-\alpha\right) \int_{\Omega}|u|^{2} d x<0 .
\end{aligned}
$$

This completes the proof. 
Lemma 3.7. If $0 \neq v \in X_{2}$, then $M_{\alpha, \beta}(v)+v$ is sign-changing.

Proof. Suppose not. Then we assume $w=M_{\alpha, \beta}(v)+v \gtreqless 0$ in $\Omega$. Let $n$ represent the Fourier coefficient of $w$ in the $\phi_{1}$ direction. We note that $n>0$ because $\int_{\Omega} w \phi_{1}>0$. Since we have maximized $J_{\alpha, \beta}$ with respect to $X_{1}$, we must have $\left\langle J_{\alpha, \beta}^{\prime}(w), \phi_{1}\right\rangle=0$. Thus

$$
0=\int_{Q}(w(x)-w(y))\left(\phi_{1}(x)-\phi_{1}(y)\right) K(x-y) d x d y-\alpha \int_{\Omega} w^{+} \phi_{1} d x+\beta \int_{\Omega} w^{-} \phi_{1} d x
$$

But $w=w^{+}$and $w^{-} \equiv 0$, so

$$
0=n \int_{Q}\left|\phi_{1}(x)-\phi_{1}(y)\right|^{2} K(x-y) d x d y-n \alpha \int_{\Omega} \phi_{1}^{2} d x=n\left(\lambda_{1}-\alpha\right) \int_{\Omega} \phi_{1}^{2} d x \neq 0,
$$

a contradiction. Hence the result holds.

In order to obtain the continuity property of $M_{\alpha, \beta}$, in the next lemma, we distinguish between the space $X_{2}$, which has the $X_{0}$ topology and $Y_{2}$, which is the set of points in $X_{2}$ endowed with the $L^{2}(\Omega)$ topology.

Lemma 3.8. $M_{\alpha, \beta}$ is locally Lipschitz continuous as a function of $\mathbb{R}^{2} \times Y_{2}$ into $X_{1}$.

Proof. Putting $u_{i}=M_{\alpha_{i}, \beta_{i}}\left(v_{i}\right)$ for $i=1,2$ into (3.1), we get

$$
\begin{aligned}
& \delta\left\|M_{\alpha_{2}, \beta_{2}}\left(v_{2}\right)-M_{\alpha_{1}, \beta_{1}}\left(v_{1}\right)\right\|^{2} \\
\leq & \left|\beta_{2}-\alpha_{2}\right|\left(\left\|M_{\alpha_{2}, \beta_{2}}\left(v_{2}\right)-M_{\alpha_{1}, \beta_{1}}\left(v_{1}\right)\right\|_{L^{2}}+\left\|v_{2}-v_{1}\right\|_{L^{2}}\right)\left\|v_{2}-v_{1}\right\|_{L^{2}} \\
& +\left|\alpha_{2}-\alpha_{1}\right|\left\|M_{\alpha_{1}, \beta_{1}}\left(v_{1}\right)\right\|_{L^{2}}\left\|M_{\alpha_{2}, \beta_{2}}\left(v_{2}\right)-M_{\alpha_{1}, \beta_{1}}\left(v_{1}\right)\right\|_{L^{2}} \\
& +\left|s_{2}-s_{1}\right|\left\|M_{\alpha_{1}, \beta_{1}}\left(v_{1}\right)+v_{1}\right\|_{L^{2}}\left\|M_{\alpha_{2}, \beta_{2}}\left(v_{2}\right)-M_{\alpha_{1}, \beta_{1}}\left(v_{1}\right)\right\|_{L^{2}} .
\end{aligned}
$$

By Poincare's inequality, we obtain

$$
\begin{aligned}
& \delta\left\|M_{\alpha_{2}, \beta_{2}}\left(v_{2}\right)-M_{\alpha_{1}, \beta_{1}}\left(v_{1}\right)\right\|^{2} \\
\leq & \left|\beta_{2}-\alpha_{2}\right|\left(\frac{1}{\lambda_{1}}\left\|M_{\alpha_{2}, \beta_{2}}\left(v_{2}\right)-M_{\alpha_{1}, \beta_{1}}\left(v_{1}\right)\right\|+\left\|v_{2}-v_{1}\right\|_{L^{2}}\right)\left\|v_{2}-v_{1}\right\|_{L^{2}} \\
& +\left|\alpha_{2}-\alpha_{1}\right|\left\|M_{\alpha_{1}, \beta_{1}}\left(v_{1}\right)\right\|_{L^{2}} \frac{1}{\lambda_{1}}\left\|M_{\alpha_{2}, \beta_{2}}\left(v_{2}\right)-M_{\alpha_{1}, \beta_{1}}\left(v_{1}\right)\right\| \\
& +\left|s_{2}-s_{1}\right|\left\|M_{\alpha_{1}, \beta_{1}}\left(v_{1}\right)+v_{1}\right\|_{L^{2}} \frac{1}{\lambda_{1}}\left\|M_{\alpha_{2}, \beta_{2}}\left(v_{2}\right)-M_{\alpha_{1}, \beta_{1}}\left(v_{1}\right)\right\| .
\end{aligned}
$$

Taking $v_{2}=v, v_{1}=0, \alpha_{1}=\alpha_{2}=\alpha$ and $\beta_{1}=\beta_{2}=\beta$. Note that $M_{\alpha, \beta}(0)=0$. Then the above inequality reduces to

$$
\delta\left\|M_{\alpha, \beta}(v)\right\|^{2} \leq|\beta-\alpha|\left(\frac{1}{\lambda_{1}}\left\|M_{\alpha, \beta}(v)\right\|+\|v\|_{L^{2}}\right)\|v\|_{L^{2}}
$$


From this inequality, one can show that $\left\|M_{\alpha, \beta}(v)\right\| \leq C\|v\|_{L^{2}}$ for an appropriate $C>0$ depending on $\delta$.

We now proceed to the main estimate. For a given $v_{1}$, we let $c_{1}=\left\|M_{\alpha_{1}, \beta_{1}}\left(v_{1}\right)\right\|_{L^{2}}$, $c_{2}=\left\|M_{\alpha_{1}, \beta_{1}}\left(v_{1}\right)+v_{1}\right\|_{L^{2}}$ and $w=\left\|M_{\alpha_{2}, \beta_{2}}\left(v_{2}\right)-M_{\alpha_{1}, \beta_{1}}\left(v_{1}\right)\right\|$. Then it follows that

$$
\delta w^{2} \leq\left(\left|\beta_{2}-\alpha_{2}\right||| v_{2}-v_{1} \|_{L^{2}}+c_{1}\left|\alpha_{2}-\alpha_{1}\right|+c_{2}\left|s_{2}-s_{1}\right|\right) \frac{1}{\lambda_{1}} w+\left|\beta_{2}-\alpha_{2}\right|\left\|v_{2}-v_{1}\right\|_{L^{2}}^{2} .
$$

Now, take $\gamma:=\left(\left|\beta_{2}-\alpha_{2}\right||| v_{2}-v_{1} \|_{L^{2}}+c_{1}\left|\alpha_{2}-\alpha_{1}\right|+c_{2}\left|s_{2}-s_{1}\right|\right)$. Then observe that $\left|\beta_{2}-\alpha_{2}\right|\left\|v_{2}-v_{1}\right\|_{L^{2}} \leq \gamma$, so

$$
\delta w^{2} \leq \frac{\gamma}{\lambda_{1}} w+\frac{\gamma^{2}}{\left|\beta_{2}-\alpha_{2}\right|} .
$$

Therefore, there exists a positive constant $K$ such that $w \leq K \gamma$ and the result follows.

Lemma 3.9. For a given $\alpha$ and $\beta, M_{\alpha, \beta}: Y_{2} \rightarrow X_{1}$ is Lipschitz continuous.

Proof. Taking $\alpha_{1}=\alpha_{2}=\alpha$ and $\beta_{1}=\beta_{2}=\beta$ in the proof of Lemma 3.8, one can easily see that $w \leq K_{1} \gamma$, where $\gamma=\left\|v_{2}-v_{1}\right\|$, and $K_{1}$ has no dependence on $c_{1}$ and $c_{2}$.

Lemma 3.10. There is a $\rho>0$ such that $\left\|M_{\alpha, \beta}(v)\right\| \leq \rho\|v\|_{L^{2}}$ for all $v \in X_{2}$.

Proof. It follows from the Lipschitz continuity and the homogeneity properties of $M_{\alpha, \beta}$.

Lemma 3.11. Suppose that $\left\{v_{k}\right\}$ is bounded in $X_{2}$, and $\left\{\alpha_{k}\right\},\left\{\beta_{k}\right\}$ are bounded sequences in $\mathbb{R}$ that satisfy our given restriction on $(\alpha, \beta)$. Then there exist subsequences, still denoted by $\left\{v_{k}\right\},\left\{\alpha_{k}\right\}$ and $\left\{\beta_{k}\right\}$ such that $\left(\alpha_{k}, \beta_{k}\right) \rightarrow(\alpha, \beta)$ in $\mathbb{R}^{2}, v_{k} \rightarrow v$ in $X_{2}, v_{k} \rightarrow v$ in $L^{2}(\Omega)$ and $M_{\alpha, \beta}\left(v_{k}\right) \rightarrow M_{\alpha, \beta}(v)$ in $X_{1}$.

Proof. The proof follows from the standard compactness arguments combined with the continuity established in Lemma 3.10 .

Lemma 3.12. If $J_{\alpha, \beta}$ has a critical point at $w=u+v$, then $u=M_{\alpha, \beta}(v)$.

Proof. $J_{\alpha, \beta}^{\prime}(w)=0$ since $J_{\alpha, \beta}$ has a critical point at $w=u+v$. Using Lemma 3.4. we have for each $v \in X_{2}, J_{\alpha, \beta}(\cdot, v)$ achieves a unique maximum. Thus $u=M_{\alpha, \beta}(v)=$ $\max _{u_{1} \in X_{1}} J_{\alpha, \beta}\left(u_{1}\right)$.

Given the last lemma, it makes sense to restrict our search for critical points to the set $\mathcal{X}_{2}:=\left\{M_{\alpha, \beta}(v)+v: v \in X_{2}\right\}$. We define $\widetilde{J}_{\alpha, \beta}: X_{2} \rightarrow \mathbb{R}$ as

$$
\widetilde{J}_{\alpha, \beta}(v)=J_{\alpha, \beta}\left(M_{\alpha, \beta}(v)+v\right) .
$$


Lemma 3.13. The functional $\widetilde{J}_{\alpha, \beta}$ is differentiable and its derivative is continuous, i.e., $\widetilde{J}_{\alpha, \beta} \in C^{1}\left(X_{2}, \mathbb{R}\right)$.

Proof. Using the maximum property and the continuity of $M_{\alpha, \beta}$, as well as the fact that $J_{\alpha, \beta}$ is $C^{1}$ on $X_{0}$, we have the following inequality

$$
\begin{aligned}
\widetilde{J}_{\alpha, \beta}\left(v_{2}\right)-\widetilde{J}_{\alpha, \beta}\left(v_{1}\right)= & J_{\alpha, \beta}\left(M_{\alpha, \beta}\left(v_{2}\right)+v_{2}\right)-J_{\alpha, \beta}\left(M_{\alpha, \beta}\left(v_{1}\right)+v_{1}\right) \\
\leq & J_{\alpha, \beta}\left(M_{\alpha, \beta}\left(v_{2}\right)+v_{2}\right)-J_{\alpha, \beta}\left(M_{\alpha, \beta}\left(v_{2}\right)+v_{1}\right) \\
= & \left\langle J_{\alpha, \beta}^{\prime}\left(M_{\alpha, \beta}\left(v_{2}\right)+v_{1}\right),\left(v_{2}-v_{1}\right)\right\rangle+o\left(\left\|v_{2}-v_{1}\right\|\right) \\
= & \left\langle J_{\alpha, \beta}^{\prime}\left(M_{\alpha, \beta}\left(v_{1}\right)+v_{1}\right),\left(v_{2}-v_{1}\right)\right\rangle+o\left(\left\|v_{2}-v_{1}\right\|\right) \\
& +\left\langle\left(J_{\alpha, \beta}^{\prime}\left(M_{\alpha, \beta}\left(v_{2}\right)+v_{1}\right)-J_{\alpha, \beta}^{\prime}\left(M_{\alpha, \beta}\left(v_{1}\right)+v_{1}\right)\right),\left(v_{2}-v_{1}\right)\right\rangle \\
= & \left\langle J_{\alpha, \beta}^{\prime}\left(M_{\alpha, \beta}\left(v_{1}\right)+v_{1}\right),\left(v_{2}-v_{1}\right)\right\rangle+o\left(\left\|v_{2}-v_{1}\right\|\right) .
\end{aligned}
$$

Similarly we can show

$$
\widetilde{J}_{\alpha, \beta}\left(v_{2}\right)-\widetilde{J}_{\alpha, \beta}\left(v_{1}\right) \geq\left\langle J_{\alpha, \beta}^{\prime}\left(M_{\alpha, \beta}\left(v_{1}\right)+v_{1}\right),\left(v_{2}-v_{1}\right)\right\rangle+o\left(\left\|v_{2}-v_{1}\right\|\right) .
$$

Hence the functional $\widetilde{J}_{\alpha, \beta}$ is continuously differentiable.

From the above Lemma 3.13, we also note the following identity

$$
\widetilde{J}_{\alpha, \beta}^{\prime}(v)=J_{\alpha, \beta}^{\prime}\left(M_{\alpha, \beta}(v)+v\right) .
$$

Lemma 3.14. $v \in X_{2}$ is a critical point of $\widetilde{J}_{\alpha, \beta}$ if and only if $M_{\alpha, \beta}(v)+v$ is a critical point of $J_{\alpha, \beta}$.

Proof. Assume that $M_{\alpha, \beta}(v)+v$ is a critical point of $J_{\alpha, \beta}$. Then $\left\langle J_{\alpha, \beta}^{\prime}\left(M_{\alpha, \beta}(v)+v\right), w\right\rangle=0$ for all $w \in X_{0}$. In particular $\left\langle J_{\alpha, \beta}^{\prime}\left(M_{\alpha, \beta}(v)+v\right), w\right\rangle=0$ for all $w \in X_{2}$. Now using equation $(3.2)$, we have $\left\langle\widetilde{J}_{\alpha, \beta}^{\prime}(v), w\right\rangle=0$ for all $w \in X_{2}$, so $v$ is a critical point of $\widetilde{J}_{\alpha, \beta}$.

Conversely, suppose that $v$ is a critical point of $\widetilde{J}_{\alpha, \beta}$. Then as above, $\left\langle J_{\alpha, \beta}^{\prime}\left(M_{\alpha, \beta}(v)+\right.\right.$ $v), w\rangle=0$ for all $w \in X_{2}$. Recall that $M_{\alpha, \beta}(v)$ maximizes $J_{\alpha, \beta}(u+v)$ for $u \in X_{1}$. Hence $\left\langle J_{\alpha, \beta}^{\prime}\left(M_{\alpha, \beta}(v)+v\right), u\right\rangle=0$ for all $u \in X_{1}$. Thus $\left\langle J_{\alpha, \beta}^{\prime}\left(M_{\alpha, \beta}(v)+v\right), w\right\rangle=0$ for all $w \in X_{0}$.

Lemma 3.15. $\widetilde{J}_{\alpha, \beta}(t v)=t^{2} \widetilde{J}_{\alpha, \beta}(v)$ for all $t \geq 0$ and for all $v \in X_{2}$.

Proof. Using the homogeneity of $J_{\alpha, \beta}$ and $M_{\alpha, \beta}$, we have

$$
\begin{aligned}
\widetilde{J}_{\alpha, \beta}(t v) & =J_{\alpha, \beta}\left(M_{\alpha, \beta}(t v)+t v\right)=J_{\alpha, \beta}\left(t M_{\alpha, \beta}(v)+t v\right) \\
& =t^{2} J_{\alpha, \beta}\left(M_{\alpha, \beta}(v)+v\right)=t^{2} \widetilde{J}_{\alpha, \beta}(v),
\end{aligned}
$$

which completes the proof. 
Now the homogeneity leads to the following lemma.

Lemma 3.16. If $v \in X_{2}$ is a critical point of $\widetilde{J}_{\alpha, \beta}$ then $\widetilde{J}_{\alpha, \beta}(v)=0$.

Proof. Differentiating $\widetilde{J}_{\alpha, \beta}(t v)=t^{2} \widetilde{J}_{\alpha, \beta}(v)$ with respect to $t$ to get $\left\langle\widetilde{J}_{\alpha, \beta}^{\prime}(t v), v\right\rangle=2 t \widetilde{J}_{\alpha, \beta}(v)$. Then the result follows by taking $t=1$.

As with $J_{\alpha, \beta}$, it is useful to think of $\widetilde{J}_{\alpha, \beta}$ as a function on $\mathbb{R}^{2} \times X_{2}$ as $\widetilde{J}_{\alpha, \beta}(v):=$ $\widetilde{J}(\alpha, \beta, v)$. Then we establish the following

Lemma 3.17. $\widetilde{J}(\alpha, \beta, \cdot):=\widetilde{J}_{\alpha, \beta}(\cdot)$ is strictly decreasing in $\alpha$ and $\beta$.

Proof. Assume that $\alpha_{1} \leq \alpha_{2}$ and $\beta_{1} \leq \beta_{2}$, where at least one of the inequality is strict. Then using the definition of $J_{\alpha, \beta}$, the fact that $M_{\alpha, \beta}(v)+v$ is sign changing and the maximizing property of $M_{\alpha, \beta}$, we obtain

$$
\begin{aligned}
\widetilde{J}\left(\alpha_{2}, \beta_{2}, v\right) & =J\left(\alpha_{2}, \beta_{2}, M\left(\alpha_{2}, \beta_{2}, v\right)+v\right) \\
& <J\left(\alpha_{1}, \beta_{1}, M\left(\alpha_{2}, \beta_{2}, v\right)+v\right) \\
& \leq J\left(\alpha_{1}, \beta_{1}, M\left(\alpha_{1}, \beta_{1}, v\right)+v\right),
\end{aligned}
$$

which completes the proof.

Lemma 3.18. Given any positive number $R$, there is a positive number $C$ such that

$$
\left|\widetilde{J}\left(\alpha_{2}, \beta_{2}, v\right)-\widetilde{J}\left(\alpha_{1}, \beta_{1}, v\right)\right| \leq C\left(\left|\alpha_{2}-\alpha_{1}\right|+\left|\beta_{2}-\beta_{1}\right|\right)
$$

whenever $\max \left\{\left|\alpha_{1}\right|,\left|\alpha_{2}\right|,\left|\beta_{1}\right|,\left|\beta_{2}\right|,\|v\|\right\} \leq R$.

Proof. Combining the Lipschitz continuity of $J_{\alpha, \beta}$ and $M_{\alpha, \beta}$ in Lemma 3.10, we obtain the desired result. We also notice that the bound is on $\|v\|$ rather than just $\|v\|_{L^{2}}$. This is because the Lipschitz constant on $J_{\alpha, \beta}$ depends on a bound in $X_{0}$.

\subsection{Minimizing in the $X_{2}$ direction}

We note that to find the critical points of $J_{\alpha, \beta}$ on $X_{0}$ has been reduced to find the critical points of $\widetilde{J}_{\alpha, \beta}$ on $X_{2}$. We know that $\widetilde{J}_{\alpha, \beta}$ is homogeneous, so it suffices to look for critical points on $\mathcal{S}_{X_{2}}:=\left\{v \in X_{2}:\|v\|_{L^{2}}=1\right\}$, a weakly closed set in $X_{0}$, i.e., if $\left\{v_{k}\right\} \subset \mathcal{S}_{X_{2}}$ and $v_{k} \rightarrow v$ weakly in $X_{0}$, then $v_{k} \rightarrow v$ strongly in $L^{2}$ so $\|v\|_{L^{2}}=1$ and $v \in \mathcal{S}_{X_{2}}$.

Lemma 3.19. $\widetilde{J}_{\alpha, \beta}$ achieves a global minimum on $\mathcal{S}_{X_{2}}$.

Proof. It is easy to see that $\widetilde{J}_{\alpha, \beta}$ is bounded below on $\mathcal{S}_{X_{2}}$. Let $\left\{v_{k}\right\} \subset \mathcal{S}_{X_{2}}$ be a minimizing sequence for $\widetilde{J}_{\alpha, \beta}$ and let $m=\inf _{v \in \mathcal{S}_{X_{2}}} \widetilde{J}_{\alpha, \beta}(v)$. Then one can easily see that $\left\|v_{k}\right\|$ is bounded. So after passing to a subsequence, we have $v_{k} \rightarrow v_{0}$ weakly in $X_{0}$ and $v_{k} \rightarrow v_{0}$ 
strongly in $L^{2}(\Omega)$ with $\left\|v_{0}\right\|_{L^{2}}=1$. By the continuity and compactness of $M_{\alpha, \beta}$, we have $M_{\alpha, \beta}\left(v_{k}\right) \rightarrow M_{\alpha, \beta}\left(v_{0}\right)$ in $X_{0}$. Using these observation as well as the weak lower semicontinuity of $X_{0}$ norm, we obtain $v_{0} \in \mathcal{S}_{X_{2}}$ such that $\widetilde{J}_{\alpha, \beta}\left(v_{0}\right)=\inf _{v \in \mathcal{S}_{X_{2}}} \widetilde{J}_{\alpha, \beta}(v)$.

If $v_{0}$ is a critical point of $\widetilde{J}_{\alpha, \beta}$ restricted to $\mathcal{S}_{X_{2}}$, then one can not conclude that it is a critical point of $\widetilde{J}_{\alpha, \beta}$ on $X_{2}$. For this, one must check the direction orthogonal to the surface $\mathcal{S}_{X_{2}}$.

Lemma 3.20. $v_{0} \in X_{2}$ is a nontrivial critical point of $\widetilde{J}_{\alpha, \beta}$ if and only if $v_{0}$ is a critical point of $\widetilde{J}_{\alpha, \beta}$ restricted to $\mathcal{S}_{X_{2}}$ and $\widetilde{J}_{\alpha, \beta}\left(v_{0}\right)=0$.

Proof. This is a standard fact for homogeneous operator, since every nontrivial element of $X_{2}$ can be written as $t v$ for some $v \in S_{X_{2}}$ and for some $t>0$. Computing derivatives separately with respect to $t$ and $v$ gives the result. For this, one can follow the proof of Lemma 3.16 .

Lemma 3.21. If $u$ is a nontrivial critical point of $J_{\alpha, \beta}$ if and only if $u=M_{\alpha, \beta}\left(v_{0}\right)+v_{0}$, where $\frac{v_{0}}{\left\|v_{0}\right\|_{L^{2}}}$ is a critical point of $\widetilde{J}_{\alpha, \beta}$ restricted to $\mathcal{S}_{X_{2}}$ and $\widetilde{J}_{\alpha, \beta}\left(v_{0}\right)=0$.

Proof. It is a direct consequence of Lemma 3.20 .

Definition 3.22. $m(\alpha, \beta):=\min _{v \in \mathcal{S}_{X_{2}}} \widetilde{J}_{\alpha, \beta}(v)$.

Lemma 3.23. $m(\alpha, \beta)$ is Lipschitz continuous and is strictly decreasing as a function of both $\alpha$ and $\beta$. Moreover, $m(\alpha, \alpha)>0$.

Proof. Let $\left(\alpha_{1}, \beta_{1}\right)$ and $\left(\alpha_{2}, \beta_{2}\right)$ be two points in the plane. Let $v_{1}$ and $v_{2}$ be the corresponding global minimizers on $\mathcal{S}_{X_{2}}$, and let $w_{i j}=M_{\alpha_{i}, \beta_{i}}\left(v_{j}\right)+v_{j}$ for $i, j=1,2$. Then using the minimizing property of $v_{i}$ and then the maximizing property of $M_{\alpha_{j}, \beta_{j}}$, we obtain

$$
\begin{aligned}
m\left(\alpha_{i}, \beta_{i}\right) & =J_{\alpha_{i}, \beta_{i}}\left(M_{\alpha_{i}, \beta_{i}}\left(v_{i}\right)+v_{i}\right) \leq J_{\alpha_{i}, \beta_{i}}\left(M_{\alpha_{i}, \beta_{i}}\left(v_{j}\right)+v_{j}\right) \\
& =J_{\alpha_{j}, \beta_{j}}\left(M_{\alpha_{i}, \beta_{i}}\left(v_{j}\right)+v_{j}\right)+\frac{1}{2}\left(\alpha_{j}-\alpha_{i}\right) \int_{\Omega}\left(w_{i j}^{+}\right)^{2}+\frac{1}{2}\left(\beta_{j}-\beta_{i}\right) \int_{\Omega}\left(w_{i j}^{-}\right)^{2} \\
& \leq J_{\alpha_{j}, \beta_{j}}\left(M_{\alpha_{j}, \beta_{j}}\left(v_{j}\right)+v_{j}\right)+\frac{1}{2}\left(\alpha_{j}-\alpha_{i}\right) \int_{\Omega}\left(w_{i j}^{+}\right)^{2}+\frac{1}{2}\left(\beta_{j}-\beta_{i}\right) \int_{\Omega}\left(w_{i j}^{-}\right)^{2} \\
& =m\left(\alpha_{j}, \beta_{j}\right)+\frac{1}{2}\left(\alpha_{j}-\alpha_{i}\right) \int_{\Omega}\left(w_{i j}^{+}\right)^{2}+\frac{1}{2}\left(\beta_{j}-\beta_{i}\right) \int_{\Omega}\left(w_{i j}^{-}\right)^{2} .
\end{aligned}
$$

Since this inequality holds for $i=1, j=2$ and $i=2, j=1$, we have

$$
\left|m\left(\alpha_{2}, \beta_{2}\right)-m\left(\alpha_{1}, \beta_{1}\right)\right| \leq c\left(\left|\alpha_{2}-\alpha_{1}\right|+\left|\beta_{2}-\beta_{1}\right|\right)
$$

where $c=\max \left\{\left\|w_{12}\right\|_{L^{2}},\left\|w_{21}\right\|_{L^{2}}\right\}$. 
Moreover, if $\alpha_{2} \geq \alpha_{1}$ and $\beta_{2} \geq \beta_{1}$ where at least one of these inequalities is strict, then $m\left(\alpha_{2}, \beta_{2}\right)<m\left(\alpha_{1}, \beta_{1}\right)$. This last conclusion uses the fact that $w_{i j}$ is sign-changing.

If $\alpha=\beta$, then for $w \in X_{0}$ we have

$$
\begin{aligned}
J_{\alpha, \beta}(w)=J_{\alpha, \alpha}(w) & =\frac{1}{2}\left(\int_{Q}|w(x)-w(y)|^{2} K(x-y) d x d y-\alpha \int_{\Omega} w^{2} d x\right) \\
& =\frac{1}{2} \sum_{j=1}^{\infty}\left(\lambda_{j}-\alpha\right) c_{j}^{2},
\end{aligned}
$$

where we are applying the Fourier decomposition of $w$. Write $w=u+v$ using the usual decomposition of $X_{0}$. Then the coefficient $\left(\lambda_{i}-\alpha\right)$ are strictly negative for $j \leq k$, so it follows that we can maximize in the $X_{1}$ direction by choosing $c_{j}=0$ for $j=1, \ldots, k$, i.e., $M_{\alpha, \beta}(v) \equiv 0$. Thus we have

$$
\widetilde{J}_{\alpha, \beta}(v)=J_{\alpha, \beta}(v)=\frac{1}{2} \sum_{j=k+1}^{\infty}\left(\lambda_{j}-\alpha\right) c_{j}^{2} .
$$

Also the coefficients $\left(\lambda_{j}-\alpha\right)$ are strictly positive and increasing for $j=k+1, k+2, \ldots$. Also $\sum_{j=k+1}^{\infty} c_{j}^{2}=\|v\|_{L^{2}}=1$. Using the Lagrange multipliers, one can show that the critical points of this sum occur when $c_{j} \equiv \pm 1$ for one $j$ and $c_{j}=0$ for all other $j$. The minimizing choice is when $c_{k+1}=1$ and $c_{j}=0$ for $j>k+1$. Hence the minimizer is $v= \pm \phi_{k+1}$ and $m(\alpha, \alpha)=\widetilde{J}_{\alpha, \beta}(v)=\frac{1}{2}\left(\lambda_{k+1}-\alpha\right)>0$.

Lemma 3.24. $m\left(\alpha, \lambda_{k+1}\right)>0$.

Proof. Let $v \in \mathcal{S}_{X_{2}}$ and let $\beta=\lambda_{k+1}$. Then using $\alpha<\lambda_{k+1}$ and $v^{+}$is nontrivial, we obtain

$$
\begin{aligned}
\widetilde{J}_{\alpha, \beta}(v) & =J_{\alpha, \beta}\left(M_{\alpha, \beta}(v)+(v)\right) \geq J_{\alpha, \beta}(v) \\
& >\frac{1}{2}\left(\int_{Q}|v(x)-v(y)|^{2} K(x-y) d x d y-\lambda_{k+1} \int_{\Omega} v^{2} d x\right) \geq 0,
\end{aligned}
$$

which follows the result.

All of the lemmas above have been leading to the following theorem.

Theorem 3.25. Assume that $\lambda_{k}<\alpha<\lambda_{k+1}$. Then one of the following is true:

(1) $m(\alpha, \beta)>0$ and $(\alpha, \beta) \notin \Sigma_{K}$ for all $\beta \geq \alpha$.

(2) There is a unique $\beta(\alpha)>\lambda_{k+1}$, such that $m(\alpha, \beta(\alpha))=0$. Moreover, $(\alpha, \beta(\alpha)) \in$ $\Sigma_{K}$, but $(\alpha, \beta) \notin \Sigma_{K}$ if $\alpha \leq \beta<\beta(\alpha)$. 
Proof. Case 1. If $\alpha \leq \beta \leq \lambda_{k+1}$ then $0<m\left(\alpha, \lambda_{k+1}\right) \leq m(\alpha, \beta)$ by Lemmas 3.24 and 3.23 . This implies $(\alpha, \beta) \notin \Sigma_{K}$ by Lemma 3.20 .

Case 2. If $\beta>\lambda_{k+1}$ then there is a $\beta(\alpha)$ such that $m(\alpha, \beta(\alpha))=0$. Clearly, one can easily see that $\beta(\alpha)$ is unique. If not, there are two $\beta_{1}(\alpha)$ and $\beta_{1}(\alpha)$ such that $m\left(\alpha, \beta_{i}(\alpha)\right)=0$ for $i=1,2$. Now $0=m\left(\alpha, \beta_{1}(\alpha)\right)<m\left(\alpha, \beta_{i}(\alpha)\right)=0$, which gives a contradiction. Since $m(\alpha, \beta(\alpha))=0$, we have $(\alpha, \beta(\alpha)) \in \Sigma_{K}$ by Lemma 3.19. Now, if $\alpha \leq \beta<\beta(\alpha)$ then $0=m(\alpha, \beta(\alpha))<m(\alpha, \beta)$ which implies $(\alpha, \beta) \notin \Sigma_{K}$.

Lemma 3.26. The curve $(\alpha, \beta(\alpha))$ is Lipschitz continuous, strictly decreasing, and contains the point $\left(\lambda_{k+1}, \lambda_{k+1}\right)$.

Proof. Consider two points $\left(\alpha_{1}, \beta_{1}\right)$ and $\left(\alpha_{2}, \beta_{2}\right)$ on $\Sigma_{K}$, characterized as above, with $\alpha_{2}>\alpha_{1}$. Let $v_{i}$ be a minimizer of $J_{\alpha_{i}, \beta_{i}}\left(M_{\alpha_{i}, \beta_{i}}(v)+v\right)$ such that $\left\|v_{i}\right\|_{L^{2}}=1$. In particular, we know that $J_{\alpha_{i}, \beta_{i}}\left(M_{\alpha_{i}, \beta_{i}}\left(v_{i}\right)+v_{i}\right)=0$ and that $J_{\alpha_{i}, \beta_{i}}\left(M_{\alpha_{i}, \beta_{i}}(v)+v\right) \geq 0$ for all $v \in X_{2}$. Let $w_{i}=M_{\alpha_{i}, \beta_{i}}\left(v_{i}\right)+v_{i}$, then we have

$$
\begin{aligned}
0 & =2 J_{\alpha_{1}, \beta_{1}}\left(w_{1}\right) \\
& =\int_{Q}\left|w_{1}(x)-w_{1}(y)\right|^{2} K(x-y) d x d y-\alpha_{1} \int_{\Omega}\left(w_{1}^{+}\right)^{2} d x-\beta_{1} \int_{\Omega}\left(w_{1}^{-}\right)^{2} d x \\
& >\int_{Q}\left|w_{1}(x)-w_{1}(y)\right|^{2} K(x-y) d x d y-\alpha_{2} \int_{\Omega}\left(w_{1}^{+}\right)^{2} d x-\beta_{1} \int_{\Omega}\left(w_{1}^{-}\right)^{2} d x,
\end{aligned}
$$

where we obtain strict inequality using the fact that $\alpha_{2}>\alpha_{1}$ and that $w_{1}$ is sign changing so that $w_{1}^{+}$is nontrivial. It follows that $m\left(\alpha_{2}, \beta_{1}\right)<0$. Since $m(\alpha, \beta)$ is strictly decreasing in $\beta$ and $m\left(\alpha_{2}, \beta_{2}\right)=0$, it must be the case that $\beta_{2}<\beta_{1}$, i.e., $\beta(\alpha)$ is strictly decreasing. Now consider

$$
\begin{aligned}
2 J_{\alpha_{2}, \beta_{1}}\left(w_{2}\right) & =\int_{Q}\left|w_{2}(x)-w_{2}(y)\right|^{2} K(x-y) d x d y-\alpha_{2} \int_{\Omega}\left(w_{2}^{+}\right)^{2}-\beta_{1} \int_{\Omega}\left(w_{2}^{-}\right)^{2} \\
& =\left(\beta_{2}-\beta_{1}\right) \int_{\Omega}\left(w_{2}^{-}\right)^{2} .
\end{aligned}
$$

It follows that $m\left(\alpha_{2}, \beta_{1}\right) \leq \frac{1}{2}\left(\beta_{2}-\beta_{1}\right) \int_{\Omega}\left(w_{2}^{-}\right)^{2}<0$. Thus

$$
\left|\beta_{2}-\beta_{1}\right| \leq 2 \frac{1}{\int_{\Omega}\left(w_{2}^{-}\right)^{2}}\left|m\left(\alpha_{2}, \beta_{1}\right)\right|=2 \frac{1}{\int_{\Omega}\left(w_{2}^{-}\right)^{2}}\left|m\left(\alpha_{2}, \beta_{1}\right)-m\left(\alpha_{2}, \beta_{2}\right)\right| .
$$

The Lipschitz estimate for $\beta(\alpha)$ follows from the Lipschitz estimate for $m(\alpha, \beta)$.

4. Nonresonance and resonance case for problem (1.1)

\subsection{The nonresonance case}

In this section we assume that $(\alpha, \beta) \in \mathbb{R}^{2}$ such that $\lambda_{k}<\alpha<\lambda_{k+1}$ and $\alpha \leq \beta<\beta(\alpha)$. By the characterization of the Fučik spectrum in Theorem 3.25 and Lemma 3.26, we 
know that $(\alpha, \beta) \notin \Sigma_{K}$. Then one should expect that (1.1) is solvable without further restrictions on either $f$ or $h$, by analogy with the Fredholm Alternative for the linear case. This is indeed the case.

For notational convenience let $E=E_{\alpha, \beta}$ and $J=J_{\alpha, \beta}$. Notice that

$$
E(u)=J(u)-\int_{\Omega}(F(u)+h u) .
$$

We will see that the geometry of $J$ dominates the geometry of $E$, so that the saddle geometry is easily proved in this case.

Lemma 4.1. There is a positive constant $K$ such that $\left|\int_{\Omega}(F(u)+h u)\right| \leq K\|u\|_{L^{2}}$ for all $u \in X_{0}$.

Proof. Since $f$ is bounded, there is an $M>0$ such that $|f(t)| \leq M$ for all $t \in \mathbb{R}$. It immediately follows that $|F(t)| \leq M|t|$ for all $t$. Thus

$$
\begin{aligned}
\left|\int_{\Omega}(F(u)+h u)\right| & \leq \int_{\Omega}|F(u)+h u| \leq \int_{\Omega}(M+|h|)|u| \\
& \leq\left(\int_{\Omega}(M+|h|)^{2}\right)^{1 / 2}\left(\int_{\Omega} u^{2}\right)^{1 / 2} .
\end{aligned}
$$

Lemma 4.2. $E$ is anticoercive when restricted to $X_{1}$.

Proof. Let $u \in X_{1}$, then using $\alpha \leq \beta, \int_{Q}|u(x)-u(y)|^{2} K(x-y) d x d y \leq \lambda_{k} \int_{\Omega} u^{2} d x$ for all $u \in X_{1}$ and Lemma 4.1, we have

$$
\begin{aligned}
E(u) & =J(u)-\int_{\Omega}(F(u)+h u) \\
& \leq\left(1-\frac{\alpha}{\lambda_{k}}\right)\|u\|^{2}+\left(\int_{\Omega}(M+|h|)^{2}\right)^{1 / 2}\left(\int_{\Omega} u^{2}\right)^{1 / 2} \\
& \leq\left(1-\frac{\alpha}{\lambda_{k}}\right)\|u\|^{2}+C\|u\| \rightarrow-\infty
\end{aligned}
$$

as $\|u\| \rightarrow \infty$, since $\lambda_{k}<\alpha$.

Lemma 4.3. The functional $E$ is bounded below and coercive on $\mathcal{X}_{2}:=\left\{M_{\alpha, \beta}(v)+v\right.$ : $\left.v \in X_{2}\right\}$.

Proof. Since $\beta<\beta(\alpha)$, we know that $\inf _{\mathcal{S}_{X_{2}}} \widetilde{J}(v) \geq c$ for some $c$. It follows that for any $v \in \mathcal{X}_{2}$

$$
J(M(v)+v)=\widetilde{J}(v)=\|v\|_{L^{2}}^{2} \widetilde{J}\left(\frac{v}{\|v\|_{L^{2}}}\right) \geq c\|v\|_{L^{2}}^{2} .
$$

Now, for $u=M(v)+v$ we have

$$
E(u) \geq c\|v\|_{L^{2}}^{2}-\left(\int_{\Omega}(M+|h|)^{2}\right)^{1 / 2}\|u\|_{L^{2}} .
$$


Recall that $\left\|M_{\alpha, \beta}(v)\right\| \leq c\|v\|_{L^{2}}$ for all $v \in X_{2}$. Then it follows that $\|u\|_{L^{2}} \leq k\|v\|_{L^{2}}$ for some $k>0$ and all $v \in X_{2}$. Thus the inequality for $E$ becomes

$$
E(u) \geq c\|v\|_{L^{2}}^{2}-k\left(\int_{\Omega}(M+|h|)^{2}\right)^{1 / 2}\|v\|_{L^{2}} .
$$

Hence one can easily conclude that $E$ is bounded below and coercive on $\mathcal{X}_{2}$.

As a result of this estimates above we can choose $R>0$ such that

$$
\sup _{u \in X_{1},\|u\|=R} E(u)<\inf _{v \in \mathcal{X}_{2}} E(v)
$$

In the next lemma we show that $\partial B_{R}(0):=\left\{x \in X_{1}:\|x\|=R\right\}$ and $\mathcal{X}_{2}$ link. Note that $\partial B_{R}(0)$ is clearly embedding of $S^{k-1}$ in $X_{0}$.

Lemma 4.4. Let $\gamma: \overline{B_{R}(0)} \subset X_{1} \rightarrow X_{0}$ be a continuous function and write $\gamma(x)=$ $\gamma_{X_{1}}(x)+\gamma_{X_{2}}(x)$, where $\gamma_{X_{1}}(x) \in X_{1}$ and $\gamma_{X_{2}}(x) \in X_{2}$. We assume that $\gamma$ fixes $\partial B_{R}$, so $\gamma_{X_{1}}(x)=x$ and $\gamma_{X_{2}}(x)=0$ for all $x \in \partial \overline{B_{R}(0)}$, then $\gamma\left(\overline{B_{R}(0)}\right) \cap \mathcal{X}_{2} \neq \emptyset$.

Proof. We must show that there is an $x \in \overline{B_{R}(0)}$ such that $\gamma_{X_{1}}(x)=M\left(\gamma_{X_{2}}(x)\right)$, so it is reasonable to study the solutions of the equation $G(x)=0$ where $G: \overline{B_{R}(0)} \rightarrow X_{1}$ : $G(x)=\gamma_{X_{1}}(x)-M\left(\gamma_{X_{2}}(x)\right)$. It is clear that $G$ is continuous. Also, if $x \in \partial \overline{B_{R}(0)}$, then $G(x)=x \neq 0$ and so the Brouwer degree $\operatorname{deg}\left(G, \overline{B_{R}(0)}, 0\right)$ is well defined. Consider the homotopy $h(t, x)=t G(x)+(1-t) x$, where $t \in[0,1]$ and $x \in \overline{B_{R}(0)}$. For $x \in \partial \overline{B_{R}(0)}$ we have $h(t, x)=t x+(1-t) x=x \neq 0$, so $\operatorname{deg}\left(G, \overline{B_{R}(0)}, 0\right)=\operatorname{deg}\left(I, \overline{B_{R}(0)}, 0\right)=1$ where $I$ represents the identity map. Hence $G(x)=0$ has a solution in $\overline{B_{R}(0)}$.

Lemma 4.5. Assume $K: \mathbb{R}^{n} \backslash\{0\} \rightarrow(0, \infty)$ satisfies assumptions (K1)-(K3), $f: \mathbb{R} \rightarrow \mathbb{R}$ is bounded and continuous, and $h \in L^{2}(\Omega)$. Let $c \in \mathbb{R}$ and let $\left\{u_{k}\right\}_{k \in \mathbb{N}}$ be a sequence in $X_{0}$ such that

$$
E\left(u_{k}\right) \leq c
$$

and

$$
\sup \left\{\left|\left\langle E^{\prime}\left(u_{k}\right), \phi\right\rangle: \phi \in X_{0},\|\phi\|=1\right|\right\} \rightarrow 0
$$

as $k \rightarrow \infty$. Then, the sequence $\left\{u_{k}\right\}_{k \in \mathbb{N}}$ is bounded in $X_{0}$. Moreover, there exists $u_{0} \in X_{0}$ such that, up to a subsequence

$$
\left\|u_{k}-u_{0}\right\| \rightarrow 0 \quad \text { as } k \rightarrow \infty
$$


Proof. Let $\left\{u_{k}\right\} \subset X_{0}$ be such that 4.2 and (4.3) hold, i.e., $E\left(u_{k}\right)$ is bounded and $E^{\prime}\left(u_{k}\right) \rightarrow 0$ in $X_{0}^{*}$. Then we show that $\left\{u_{k}\right\}$ is bounded in $X_{0}$. Suppose by contradiction that $\left\|u_{k}\right\|_{L^{2}}$ is unbounded. Then without loss of generality we may assume that $\left\|u_{k}\right\|_{L^{2}}$ is increasing to $\infty$. Consider $v_{k}:=\frac{u_{k}}{\left\|u_{k}\right\|_{L^{2}}}$. Then,

$$
\begin{aligned}
\frac{E\left(u_{k}\right)}{\left\|u_{k}\right\|_{L^{2}}^{2}}= & \frac{1}{2} \int_{Q}\left|v_{k}(x)-v_{k}(y)\right|^{2} K(x-y) d x d y-\frac{\alpha}{2} \int_{\Omega}\left(v_{k}^{+}\right)^{2}-\frac{\beta}{2} \int_{\Omega}\left(v_{k}^{-}\right)^{2} \\
& -\frac{1}{\left\|u_{k}\right\|_{L^{2}}^{2}} \int_{\Omega}\left(F\left(u_{k}\right)+h u_{k}\right) .
\end{aligned}
$$

Now equation 4.2 implies that $\frac{E\left(u_{k}\right)}{\left\|u_{k}\right\|_{L^{2}}^{2}} \rightarrow 0$. Also $\frac{\alpha}{2} \int_{\Omega}\left(v_{k}^{+}\right)^{2}+\frac{\beta}{2} \int_{\Omega}\left(v_{k}^{-}\right)^{2}+\frac{1}{\left\|u_{k}\right\|_{L^{2}}^{2}} \int_{\Omega}\left(F\left(u_{k}\right)\right.$ $+h u_{k}$ ) is bounded. Then it follows that $\left\{v_{k}\right\}$ is bounded in $X_{0}$, a reflexive space (being a Hilbert space), so up to a subsequence, there exists $v_{0} \in X_{0}$ such that $v_{k} \rightarrow v_{0}$ weakly in $X_{0}, v_{k} \rightarrow v_{0}$ strongly in $L^{2}(\Omega)$ and $\left\|v_{0}\right\|_{L^{2}}=1$. Now for any $w \in X_{0}$, we consider

$$
\begin{aligned}
\left\langle\frac{E^{\prime}\left(u_{k}\right)}{\left\|u_{k}\right\|_{L^{2}}}, w\right\rangle= & \int_{Q}\left(v_{k}(x)-v_{k}(y)\right)(w(x)-w(y)) K(x-y) d x d y-\alpha \int_{\Omega}\left(v_{k}^{+}\right) w \\
& +\beta \int_{\Omega}\left(v_{k}^{-}\right) w-\frac{1}{\left\|u_{k}\right\|_{L^{2}}}\left(\int_{\Omega}\left(f\left(u_{k}\right)+h\right) w\right) .
\end{aligned}
$$

Using the boundedness of $f$ it is clear that $\frac{1}{\left\|u_{k}\right\|_{L^{2}}} \int_{\Omega}\left(f\left(u_{k}\right)+h\right) w \rightarrow 0$. Also using the $L^{2}$ convergence of $v_{k}$, it is clear that $v_{k}^{+}$and $v_{k}^{-}$converges to $v_{0}^{+}$and $v_{0}^{-}$respectively in $L^{2}$. So,

$$
-\alpha \int_{\Omega}\left(v_{k}^{+}\right) w+\beta \int_{\Omega}\left(v_{k}^{-}\right) w \rightarrow-\alpha \int_{\Omega}\left(v_{0}^{+}\right) w+\beta \int_{\Omega}\left(v_{0}^{-}\right) w .
$$

By the weak convergence of $v_{k}$ in $X_{0}$, we have for every $\phi \in X_{0}$,

$$
\begin{aligned}
& \int_{Q}\left(v_{k}(x)-v_{k}(y)\right)(\phi(x)-\phi(y)) K(x-y) d x d y \\
\rightarrow & \int_{Q}\left(v_{0}(x)-v_{0}(y)\right)(\phi(x)-\phi(y)) K(x-y) d x d y
\end{aligned}
$$

as $k \rightarrow \infty$. Thus using the above discussion, we obtain $\left\langle\frac{E^{\prime}\left(u_{k}\right)}{\left\|u_{k}\right\|_{L^{2}}}, w\right\rangle \rightarrow 0$. Hence

$$
0=\int_{Q}\left(v_{0}(x)-v_{0}(y)\right)(w(x)-w(y)) d x d y-\alpha \int_{\Omega}\left(v_{0}^{+}\right) w+\beta \int_{\Omega}\left(v_{0}^{-}\right) w \quad \forall w \in X_{0} .
$$

Therefore $v_{0}$ is a nontrivial weak solution of $(1.2)$. This contradicts the fact that $(\alpha, \beta) \notin$ $\Sigma_{K}$. Hence $\left\{u_{k}\right\}$ is bounded in $L^{2}$. Now

$$
E\left(u_{k}\right)=\frac{1}{2}\left\|u_{k}\right\|^{2}-\frac{\alpha}{2} \int_{\Omega}\left(u_{k}^{+}\right)^{2}-\frac{\beta}{2} \int_{\Omega}\left(u_{k}^{-}\right)^{2}-\int_{\Omega}\left(F\left(u_{k}\right)+h u_{k}\right) .
$$

We see that $E\left(u_{k}\right), \int_{\Omega}\left(u_{k}^{+}\right)^{2}, \int_{\Omega}\left(u_{k}^{-}\right)^{2}$ and $\int_{\Omega}\left(F\left(u_{k}\right)+h u_{k}\right)$ are all bounded, so $\left\|u_{k}\right\|$ must be bounded. 
Since $\left\{u_{k}\right\}_{k \in \mathbb{N}}$ is a bounded sequence in $X_{0}$, there exists $u_{0} \in X_{0}$ such that up to a subsequence $u_{k}$ converges to $u_{0}$ weakly in $X_{0}$, i.e., for every $\phi \in X_{0}$,

$$
\begin{aligned}
& \int_{Q}\left(u_{k}(x)-u_{k}(y)\right)(\phi(x)-\phi(y)) K(x-y) d x d y \\
\rightarrow & \int_{Q}\left(u_{0}(x)-u_{0}(y)\right)(\phi(x)-\phi(y)) K(x-y) d x d y
\end{aligned}
$$

as $k \rightarrow \infty$. Moreover, $u_{k} \rightarrow u_{0}$ strongly in $L^{\mu}(\Omega)$ for any $\mu \in\left[1,2_{s}^{*}\right)$ and $u_{k}(x) \rightarrow u_{0}(x)$ a.e. in $\mathbb{R}^{n}$ as $k \rightarrow \infty$. Now,

$$
\begin{aligned}
& \left\langle E^{\prime}\left(u_{k}\right),\left(u_{k}-u_{0}\right)\right\rangle \\
= & \int_{Q}\left(u_{k}(x)-u_{k}(y)\right)\left(\left(u_{k}-u_{0}\right)(x)-\left(u_{k}-u_{0}\right)(y)\right) K(x-y) d x d y \\
& -\alpha \int_{\Omega}\left(u_{k}^{+}\right)\left(u_{k}-u_{0}\right)+\beta \int_{\Omega}\left(u_{k}^{-}\right)\left(u_{k}-u_{0}\right)-\int_{\Omega}\left(f\left(u_{k}\right)+h\right)\left(u_{k}-u_{0}\right) .
\end{aligned}
$$

Also using the $L^{2}$ boundedness of $u_{k}^{+}, u_{k}^{-}$, and $f\left(u_{k}\right)+h$ and the fact that $u_{k} \rightarrow u_{0}$ strongly in $L^{2}$, we obtain

$$
-\alpha \int_{\Omega}\left(u_{k}^{+}\right)\left(u_{k}-u_{0}\right)+\beta \int_{\Omega}\left(u_{k}^{-}\right)\left(u_{k}-u_{0}\right)-\int_{\Omega}\left(f\left(u_{k}\right)+h\right)\left(u_{k}-u_{0}\right) \rightarrow 0 .
$$

From (4.3), we have $\left\langle E^{\prime}\left(u_{k}\right),\left(u_{k}-u_{0}\right)\right\rangle \rightarrow 0$. Thus using this, 4.4) and (4.5), we obtain

$$
\int_{Q}\left(u_{k}(x)-u_{k}(y)\right)\left(\left(u_{k}-u_{0}\right)(x)-\left(u_{k}-u_{0}\right)(y)\right) K(x-y) d x d y \rightarrow 0 \quad \text { as } k \rightarrow \infty .
$$

Hence, using this and the weak convergence of $u_{k}$, we obtain

$$
\int_{Q}\left|u_{k}(x)-u_{k}(y)\right|^{2} K(x-y) d x d y \rightarrow \int_{Q}\left|u_{0}(x)-u_{0}(y)\right|^{2} K(x-y) d x d y \quad \text { as } k \rightarrow \infty .
$$

It follows that $u_{k} \rightarrow u_{0}$ strongly in $X_{0}$.

Proof of Theorem 1.3. By the saddle point theorem we can now conclude the proof.

\subsection{The resonance case}

In this section, we study the problem (1.1) in the presence of a resonance, namely when $(\alpha, \beta) \in \mathbb{R}^{2}$ is an element of Fučik spectrum. This kind of problem is harder to solve than the nonresonant one and we have to impose further conditions on the nonlinearities. We assume that $\beta=\beta(\alpha)$. Many of the argument from the previous section are still applicable. Two notable exceptions are establishing a lower bound for $E$ on $\mathcal{X}_{2}$ and proving (PS). Since this case is analogous to the case $\lambda=\lambda_{k+1}$ in this Fredholm Alternative, we should expect that the solutions will only exist if a generalized orthogonality condition is satisfied. Such 
conditions were first studied in 70s and known as Landesman-Lazer conditions [13]. We will assume (1.5): $\lim _{k \rightarrow \infty} \int_{\Omega}\left(F\left(u_{k}\right)+h u_{k}\right) d x=-\infty$, a generalized Landesman-Lazer condition.

Lemma 4.6. If (1.5) is satisfied, then $E$ define in (4.1) is bounded below on $\mathcal{X}_{2}$.

Proof. Suppose that $\left\{u_{k}\right\} \subset \mathcal{X}_{2}$ such that $E\left(u_{k}\right) \rightarrow-\infty$. We write $u_{k}=M_{\alpha, \beta}\left(v_{k}\right)+v_{k}$. Then arguments similar to those in the proof of Lemma 4.3 , we see that no subsequence of $\left\{u_{k}\right\}$ lies in a set of the form $\left\{u \in \mathcal{X}_{2}: u=M_{\alpha, \beta}(v)+v, \widetilde{J}_{\alpha, \beta}(v) \geq c\|v\|_{L^{2}}^{2}\right\}$, where $c>0$. Thus $\widetilde{J}_{\alpha, \beta}\left(\frac{v_{k}}{\left\|v_{k}\right\|_{L^{2}}}\right) \rightarrow 0$ and $\frac{v_{k}}{\left\|v_{k}\right\|_{L^{2}}}$ must be a minimizing sequence of $\widetilde{J}_{\alpha, \beta}$. Similarly, using the same arguments in the proof of Lemma 3.19 one can note that $\frac{v_{k}}{\left\|v_{k}\right\|_{L^{2}}} \rightarrow v_{0}$ weakly in $X_{0}$ and $\frac{v_{k}}{\left\|v_{k}\right\|_{L^{2}}} \rightarrow v_{0}$ strongly in $L^{2}(\Omega)$. This implies that $\frac{u_{k}}{\left\|u_{k}\right\|_{L^{2}}} \rightarrow \phi$ weakly in $X_{0}$ and $\frac{u_{k}}{\left\|u_{k}\right\|_{L^{2}}} \rightarrow \phi$ strongly in $L^{2}(\Omega)$, where $\phi=M_{\alpha, \beta}(v)+v$ is a nontrivial eigenfunction associated with $(\alpha, \beta)$. By $(1.5)$, we know that $\lim _{k \rightarrow \infty} \int_{\Omega}\left(F\left(u_{k}\right)+h u_{k}\right)=-\infty$ and it immediately follows that $E\left(u_{k}\right) \rightarrow \infty$, a contradiction. Hence $E$ is bounded below on $\mathcal{X}_{1}$.

Lemma 4.7. Assume $K: \mathbb{R}^{n} \backslash\{0\} \rightarrow(0, \infty)$ satisfies assumptions (K1)-(K3), $f$ is a bounded and continuous function and $h \in L^{2}(\Omega)$. Let $\left\{u_{k}\right\}_{k \in \mathbb{N}}$ be a sequence in $X_{0}$ such that (4.2) and (4.3) hold. Then, the sequence $\left\{u_{k}\right\}_{k \in \mathbb{N}}$ is bounded in $X_{0}$ if (1.5) is satisfied.

Proof. The first part of the proof is identical the argument in the proof of Lemma 4.5. We start with the hypothetical sequence $\left\{u_{k}\right\}$ such that $(4.2)$ and $(4.3)$ hold. Suppose $\left\|u_{k}\right\|_{L^{2}}$ is unbounded. Then argue up to the point, where we have $v_{k} \rightarrow v_{0}$ weakly in $X_{0}, v_{k} \rightarrow v_{0}$ strongly in $L^{2}(\Omega)$, where $\left\|v_{0}\right\|_{L^{2}}=1$ and $v_{0}$ is an eigenfunction associated with $(\alpha, \beta)$. Of course, in the resonance case this is not yet a contradiction, so a further argument is needed.

Write $u_{k}=w_{k}+v_{k}=\widetilde{w}_{k}+M_{\alpha, \beta}\left(v_{k}\right)+v_{k}$. Now using the fact that $\left\langle J_{\alpha, \beta}^{\prime}\left(M_{\alpha, \beta}\left(v_{k}\right)+\right.\right.$ $\left.\left.v_{k}\right), u\right\rangle=0$ for all $u \in X_{1}$ and Lemma 3.1, we have

$$
\begin{aligned}
\left\langle E^{\prime}\left(u_{k}\right), \widetilde{w}_{k}\right\rangle= & \left\langle J_{\alpha, \beta}^{\prime}\left(u_{k}\right), \widetilde{w}_{k}\right\rangle-\int_{\Omega}\left(f\left(u_{k}\right)+h\right) \widetilde{w}_{k} \\
= & \left\langle J_{\alpha, \beta}^{\prime}\left(\widetilde{w}_{k}+M_{\alpha, \beta}\left(v_{k}\right)+v_{k}\right), \widetilde{w}_{k}\right\rangle-\int_{\Omega}\left(f\left(u_{k}\right)+h\right) \widetilde{w}_{k} \\
= & \left\langle J_{\alpha, \beta}^{\prime}\left(\widetilde{w}_{k}+M_{\alpha, \beta}\left(v_{k}\right)+v_{k}\right), \widetilde{w}_{k}\right\rangle-\left\langle J_{\alpha, \beta}^{\prime}\left(M_{\alpha, \beta}\left(v_{k}\right)+v_{k}\right), \widetilde{w}_{k}\right\rangle \\
& -\int_{\Omega}\left(f\left(u_{k}\right)+h\right) \widetilde{w}_{k} \\
\leq & -\delta\left\|\widetilde{w}_{k}\right\|_{L^{2}}^{2}-\int_{\Omega}\left(f\left(u_{k}\right)+h\right) \widetilde{w}_{k} .
\end{aligned}
$$

It follows that $\widetilde{w}_{k}$ is bounded. Note that $\left\langle J_{\alpha, \beta}^{\prime}\left(u_{k}\right), \widetilde{w}_{k}\right\rangle$ must also be bounded. 
Now consider

$$
\begin{aligned}
E\left(u_{k}\right) & =J_{\alpha, \beta}\left(u_{k}\right)-\int_{\Omega}\left(F\left(u_{k}\right)+h u_{k}\right) \\
& \geq J_{\alpha, \beta}\left(\widetilde{w}_{k}+M_{\alpha, \beta}\left(v_{k}\right)+v_{k}\right)-J_{\alpha, \beta}\left(M_{\alpha, \beta}\left(v_{k}\right)+v_{k}\right)-\int_{\Omega}\left(F\left(u_{k}\right)+h u_{k}\right),
\end{aligned}
$$

because $J_{\alpha, \beta}\left(M_{\alpha, \beta}\left(v_{k}\right)+v_{k}\right) \geq 0$. Let $g(t)=J_{\alpha, \beta}\left(M_{\alpha, \beta}\left(v_{k}\right)+v_{k}+t \widetilde{w}_{k}\right)$. It follows from the properties of $J_{\alpha, \beta}$ that $g^{\prime}(0)=0$ and $g^{\prime}(t)$ is decreasing. By the Mean value Theorem $g(1)-g(0)=g^{\prime}(c)$, for some $c \in(0,1)$. Hence $g(1)-g(0) \geq g^{\prime}(1)$. It follows that

$$
J_{\alpha, \beta}\left(\widetilde{w}_{k}+M_{\alpha, \beta}\left(v_{k}\right)+v_{k}\right)-J_{\alpha, \beta}\left(M_{\alpha, \beta}\left(v_{k}\right)+v_{k}\right) \geq\left\langle J_{\alpha, \beta}^{\prime}\left(u_{k}\right), \widetilde{w}_{k}\right\rangle
$$

and thus

$$
E\left(u_{k}\right) \geq\left\langle J_{\alpha, \beta}^{\prime}\left(u_{k}\right), \widetilde{w}_{k}\right\rangle-\int_{\Omega}\left(F\left(u_{k}\right)+h u_{k}\right) .
$$

But the first term on the right-hand side is bounded and the second goes to $-\infty$ by 1.5 . This contradicts the assumption that $E\left(u_{k}\right)$ is bounded. Hence $\left\{u_{k}\right\}$ is bounded in $L^{2}(\Omega)$, the remaining proof follows exactly as in the proof of Lemma 4.5 .

Proof of Theorem 1.4. One can conclude the proof from Lemmas 4.6, 4.7 and saddle point theorem.

\section{Acknowledgments}

The author's research is partially supported by National Board for Higher Mathematics, Government of India, grant number: 2/40(2)/2015/R\&D-II/5488 and Science and Engineering Research Board, Government of India, Grant number: ECR/2017/002651.

\section{References}

[1] C. Bucur and E. Valdinoci, Nonlocal Diffusion and Applications, Lecture Notes of the Unione Matematica Italiana 20, Springer, Bologna, 2016.

[2] L. A. Caffarelli, S. Salsa and L. Silvestre, Regularity estimates for the solution and the free boundary of the obstacle problem for the fractional Laplacian, Invent. Math. 171 (2008), no. 2, 425-461.

[3] A. Castro, Hammerstein integral equations with indefinite kernel, Internat. J. Math. Math. Sci. 1 (1978), no. 2, 187-201.

[4] A. Castro and C. Chang, A variational characterization of the Fucik spectrum and applications, Rev. Colombiana Mat. 44 (2010), no. 1, 23-40. 
[5] M. Cuesta, D. de Figueiredo and J.-P. Gossez, The beginning of the Fučik spectrum for the p-Laplacian, J. Differential Equations 159 (1999), no. 1, 212-238.

[6] E. Di Nezza, G. Palatucci and E. Valdinoci, Hitchhiker's guide to the fractional Sobolev spaces, Bull. Sci. Math. 136 (2012), no. 5, 521-573.

[7] P. Drábek and S. B. Robinson, On the Fredholm alternative for the Fučík spectrum, Abstr. Appl. Anal. 2010 (2010), Art. ID 125464, 20 pp.

[8] - On the solvability of resonance problems with respect to the Fučik spectrum, J. Math. Anal. Appl. 418 (2014), no. 2, 884-905.

[9] M. M. Fall and V. Felli, Unique continuation property and local asymptotics of solutions to fractional elliptic equations, Comm. Partial Differential Equations 39 (2014), no. $2,354-397$.

[10] D. de Figueiredo and J.-P. Gossez, On the first curve of the Fučik spectrum of an elliptic operator, Differential Integral Equations 7 (1994), no. 5-6, 1285-1302.

[11] A. Fiscella, R. Servadei and E. Valdinoci, Asymptotically linear problems driven by fractional Laplacian operators, Math. Methods Appli. Sci. 38 (2015), no. 16, 35513563.

[12] S. Goyal and K. Sreenadh, On the Fučik spectrum of non-local elliptic operators, NoDEA Nonlinear Differential equations Appl. 21 (2014), no. 4, 567-588.

[13] E. M. Landesman and A. C. Lazer, Nonlinear perturbations of linear elliptic boundary value problems at resonance, J. Math. Mech. 19 (1969/1970), 609-623.

[14] E. Massa, On a variational characterization of the Fučik spectrum of the Laplacian and a superlinear Sturm-Liouville equation, Proc. Roy. Soc. Edinburgh Sect. A 134 (2004), no. 3, 557-577.

[15] R. Servadei and E. Valdinoci, Mountain pass solutions for non-local elliptic operators, J. Math. Anal. Appl. 389 (2012), no. 2, 887-898.

[16] Variational methods for non-local operators of elliptic type, Discrete Contin. Dyn. Syst. 33 (2013), no. 5, 2105-2137.

[17] Weak and viscosity solutions of the fractional Laplace equation, Publ. Mat. 58 (2014), no. 1, 133-154.

[18] L. Silvestre, Regularity of the obstacle problem for a fractional power of the Laplace operator, Comm. Pure Appl. Math. 60 (2007), no. 1, 67-112. 
Sarika Goyal

Department of Mathematics, Bennett University, Greater Noida, Uttar Pradesh, India E-mail address: sarika1.iitd@gmail.com, sarika.goyal@bennet.edu.in 